\title{
Compact Proofs of Retrievability
}

\author{
Hovav Shacham* \\ hovav@cs.ucsd.edu
}

\author{
Brent Waters ${ }^{\dagger}$ \\ bwaters@cs.utexas .edu
}

\begin{abstract}
In a proof-of-retrievability system, a data storage center must prove to a verifier that he is actually storing all of a client's data. The central challenge is to build systems that are both efficient and provably secure - that is, it should be possible to extract the client's data from any prover that passes a verification check. In this paper, we give the first proof-of-retrievability schemes with full proofs of security against arbitrary adversaries in the strongest model, that of Juels and Kaliski. Our first scheme, built from BLS signatures and secure in the random oracle model, has the shortest query and response of any proof-of-retrievability with public verifiability. Our second scheme, which builds elegantly on pseudorandom functions (PRFs) and is secure in the standard model, has the shortest response of any proof-of-retrievability scheme with private verifiability (but a longer query). Both schemes rely on homomorphic properties to aggregate a proof into one small authenticator value.
\end{abstract}

\section{Introduction}

In this paper, we give the first proof-of-retrievability schemes with full proofs of security against arbitrary adversaries in the Juels-Kaliski model. Our first scheme has the shortest query and response of any proof-of-retrievability with public verifiability and is secure in the random oracle model. Our second scheme has the shortest response of any proof-of-retrievability scheme with private verifiability (but a longer query), and is secure in the standard model.

Proofs of storage. Recent visions of "cloud computing" and "software as a service" call for data, both personal and business, to be stored by third parties, but deployment has lagged. Users of outsourced storage are at the mercy of their storage providers for the continued availability of their data. Even Amazon's S3, the best-known storage service, has recently experienced significant downtime 1

In an attempt to aid the deployment of outsourced storage, cryptographers have designed systems that would allow users to verify that their data is still available and ready for retrieval if needed: Deswarte, Quisquater, and Saïdane [15], Gazzoni Filho and Barreto [17], and Schwarz and Miller [28]. In these systems, the client and server engage in a protocol; the client seeks to be convinced by the protocol interaction that his file is being stored. Such a capability can be important

\footnotetext{
* Supported in part by a MURI grant administered by the Air Force Office of Scientific Research.

${ }^{\dagger}$ Supported by NSF CNS-0749931, CNS-0524252, CNS-0716199; the US Army Research Office under the CyberTA Grant No. W911NF-06-1-0316; and the U.S. Department of Homeland Security under Grant Award Number 2006CS-001-000001.

${ }^{1}$ See, e.g., http://blogs.zdnet.com/projectfailures/?p=602
} 
to storage providers as well. Users may be reluctant to entrust their data to an unknown startup; an auditing mechanism can reassure them that their data is indeed still available.

Evaluation: formal security models. Such proof-of-storage systems should be evaluated by both "systems" and "crypto" criteria. Systems criteria include: (1) the system should be as efficient as possible in terms of both computational complexity and communication complexity of the proofof-storage protocol, and the storage overhead on the server should be as small as possible; (2) the system should allow unbounded use rather than imposing a priori bound on the number of auditprotocol interactions 2 (3) verifiers should be stateless, and not need to maintain and update state between audits, since such state is difficult to maintain if the verifier's machine crashes or if the verifier's role is delegated to third parties or distributed among multiple machine ${ }^{3}$ Statelessness and unbounded use are required for proof-of-storage systems with public verifiability, in which anyone can undertake the role of verifier in the proof-of-storage protocol, not just the user who originally stored the file 4

The most important crypto criterion is this: Whether the protocol actually establishes that any server that passes a verification check for a file - even a malicious server that exhibits arbitrary, Byzantine behavior - is actually storing the file. The early cryptographic papers lacked a formal security model, let alone proofs. But provable security matters. Even reasonable-looking protocols could in fact be insecure; see Appendix B for an example.

The first papers to consider formal models for proofs of storage were by Naor and Rothblum, for "authenticators" [25], and by Juels and Kaliski, for "proofs of retrievability" [20]. Though the details of the two models are different, the insight behind both is the same: in a secure system if a server can pass an audit then a special extractor algorithm, interacting with the server, must be able (w.h.p.) to extract the file. ${ }^{5}$

A simple MAC-based construction. In addition, the Naor-Rothblum and Juels-Kaliski papers describe similar proof-of-retrievability protocols. The insight behind both is that checking that most of a file is stored is easier than checking that all is. If the file to be stored is first encoded redundantly, and each block of the encoded file is authenticated using a MAC, then it is sufficient for the client to retrieve a few blocks together with their MACs and check, using his secret key, that these blocks are correct. Naor and Rothblum prove their scheme secure in their model ${ }^{6}$ The simple protocol obtained here uses techniques similar to those proposed by Lillibridge et al. [22]. Signatures can be used instead of MACs to obtain public verifiability.

The downside to this simple solution is that the server's response consists of $\lambda$ block-authenticator pairs, where $\lambda$ is the security parameter. If each authenticator is $\lambda$ bits long, as required in the

\footnotetext{
${ }^{2}$ We believe that systems allowing a bounded number of interactions can be useful, but only as stepping stones towards fully secure systems. Some examples are bounded identity-based encryption [19] and bounded CCA-secure encryption 12]; in these systems, security is maintained only as long as the adversary makes at most $t$ private key extraction or decryption queries.

${ }^{3}$ We note that the sentinel-based scheme of Juels and Kaliski [20], the scheme of Ateniese, Di Pietro, Mancini, and Tsudik [5], and the scheme of Shah, Swaminathan and Baker [29] lack both unbounded use and statelessness. We do not consider these schemes further in this paper.

${ }^{4}$ Ateniese et al. 3] were the first to consider public verifiability for proof-of-storage schemes.

${ }^{5}$ This is, of course, similar to the intuition behind proofs of knowledge.

${ }^{6}$ Juels and Kaliski do not give a proof of security against arbitrary adversaries, but this proof is trivial using the techniques we develop in this paper; for completeness, we give the proof in Section 5 .
} 
Juels-Kaliski model, then the response is $\lambda^{2} \cdot(s+1)$ bits, where the ratio of file block to authenticator length is $s: 1,7$

Homomorphic authenticators. Ateniese et al. [3] describe a proof-of-storage scheme that improves on the response length of the simple MAC-based scheme using homomorphic authenticators. In their scheme, the authenticators $\sigma_{i}$ on each file block $m_{i}$ are constructed in such a way that a verifier can be convinced that a linear combination of blocks $\sum_{i} \nu_{i} m_{i}$ (with arbitrary weights $\left\{\nu_{i}\right\}$ ) was correctly generated using an authenticator computed from $\left\{\sigma_{i}\right\}$ \&

When using homomorphic authenticators, the server can combine the blocks and $\lambda$ authenticators in its response into a single aggregate block and authenticator, reducing the response length by a factor of $\lambda$. As an additional benefit, the Ateniese et al. scheme is the first with public verifiability. The homomorphic authenticators of Ateniese et al. are based on RSA and are thus relatively long.

Unfortunately, Ateniese et al. do not give a rigorous proof of security for their scheme. In particular, they do not show that one can extract a file (or even a significant fraction of one) from a prover that is able to answer auditing queries convincingly. The need for rigor in extraction arguments applies equally to both the proof-of-retrievability model we consider and the weaker proof of data possession model considered by Ateniese et al $?^{9}$

Our contributions. In this paper, we make two contributions.

1. We describe two new short, efficient homomorphic authenticators. The first, based on PRFs, gives a proof-of-retrievability scheme secure in the standard model. The second, based on BLS signatures [8], gives a proof-of-retrievability scheme with public verifiability secure in the random oracle model.

2. We prove both of the resulting schemes secure in a variant of the Juels-Kaliski model. Our schemes are the first with a security proof against arbitrary adversaries in this model.

The scheme with public retrievability has the shortest query and response of any proof-of-retrievability scheme: 20 bytes and 40 bytes, respectively, at the 80 -bit security level. The scheme with private retrievability has the shortest response of any proof-of-retrievability scheme (20 bytes), matching the response length of the Naor-Rothblum scheme in a more stringent security model, albeit at the cost of a longer query. We believe that derandomizing the query in this scheme is the major remaining open problem for proofs of retrievability.

\subsection{Our Schemes}

In our schemes, as in the Juels-Kaliski scheme, the user breaks an erasure encoded file into $n$ blocks $m_{1}, \ldots, m_{n} \in Z_{p}$ for some large prime $p$. The erasure code should allow decoding in the presence of adversarial erasure. Erasure codes derived from Reed-Solomon codes have this property, but decoding and encoding are slow for large files. In Appendix $\mathrm{A}$ we discuss how to make use of more efficient codes secure only against random erasures.

\footnotetext{
${ }^{7}$ Naor and Rothblum show that one-bit MACs suffice for proving security in their less stringent model, for an overall response length of $\lambda \cdot(s+1)$ bits. The Naor-Rothblum scheme is not secure in the Juels-Kaliski model.

${ }^{8}$ In the Ateniese et al. construction the aggregate authenticator is $\prod_{i} \sigma_{i}^{\nu_{i}} \bmod N$.

${ }^{9}$ For completeness, we give a correct and fully proven Ateniese-et-al.-inspired, RSA-based scheme, together with a full proof of security, in Section 6
} 
The user authenticates each block as follows. She chooses a random $\alpha \in \mathbb{Z}_{p}$ and PRF key $k$ for function $f$. These values serve as her secret key. She calculates an authentication value for each block $i$ as

$$
\sigma_{i}=f_{k}(i)+\alpha m_{i} \in \mathbb{Z}_{p} .
$$

The blocks $\left\{m_{i}\right\}$ and authenticators $\left\{\sigma_{i}\right\}$ are stored on the server. The proof of retrievability protocol is as follows. The verifier chooses a random challenge set $I$ of $l$ indices along with $l$ random coefficients in $Z_{p}{ }^{10}$ Let $Q$ be the set $\left\{\left(i, \nu_{i}\right)\right\}$ of challenge index-coefficient pairs. The verifier sends $Q$ to the prover. The prover then calculates the response, a pair $(\sigma, \mu)$, as

$$
\sigma \leftarrow \sum_{\left(i, \nu_{i}\right) \in Q} \nu_{i} \cdot \sigma_{i} \quad \text { and } \quad \mu \leftarrow \sum_{\left(i, \nu_{i}\right) \in Q} \nu_{i} \cdot m_{i}
$$

Now verifier can check that the response was correctly formed by checking that

$$
\sigma \stackrel{?}{=} \alpha \cdot \mu+\sum_{\left(i, \nu_{i}\right) \in Q} \nu_{i} \cdot f_{k}(i)
$$

It is clear that our techniques admit short responses. But it is not clear that our new system admits a simulator that can extract files. Proving that it does is quite challenging, as we discuss below. In fact, unlike similar, seemingly correct schemes (see Appendix B), our scheme is provably secure in the standard model.

A scheme with public verifiability. Our second scheme is publicly verifiable. It follows the same framework as the first, but instead uses BLS signatures [8] for authentication values that can be publicly verified. The structure of these signatures allows for them to be aggregated into linear combinations as above. We prove the security of this scheme under the Computational Diffie-Hellman assumption over bilinear groups in the random oracle model.

Let $e: G \times G \rightarrow G_{T}$ be a computable bilinear map with group $G$ 's support being $\mathbb{Z}_{p}$. A user's private key is $x \in \mathbb{Z}_{p}$, and her public key is $v=g^{x} \in G$ along with another generator $u \in G$. The signature on block $i$ is $\sigma_{i}=\left[H(i) u^{m_{i}}\right]^{x}$. On receiving query $Q=\left\{\left(i, \nu_{i}\right)\right\}$, the prover computes and sends back $\sigma \leftarrow \prod_{\left(i, \nu_{i}\right) \in Q} \sigma_{i}^{\nu_{i}}$ and $\mu \leftarrow \sum_{\left(i, \nu_{i}\right) \in Q} \nu_{i} \cdot m_{i}$. The verification equation is:

$$
e(\sigma, g) \stackrel{?}{=} e\left(\prod_{\left(i, \nu_{i}\right) \in Q} H(i)^{\nu_{i}} \cdot u^{\mu}, v\right)
$$

This scheme has public verifiability: the private key $x$ is required for generating the authenticators $\left\{\sigma_{i}\right\}$ but the public key $v$ is sufficient for the verifier in the proof-of-retrievability protocol.

Parameter selection. Let $\lambda$ be the security parameter; typically, $\lambda=80$. For the scheme with private verification, $p$ should be a $\lambda$ bit prime. For the scheme with public verification, $p$ should be a $2 \lambda$-bit prime, and the curve should be chosen so that discrete logarithm is $2^{\lambda}$-secure. For values of $\lambda$ up to 128, Barreto-Naehrig curves [6] are the right choice; see the survey by Freeman, Scott, and Teske [16].

Let $n$ be the number of blocks in the file. We assume that $n \gg \lambda$. Suppose we use a rate- $\rho$ erasure code, i.e., one in which any $\rho$-fraction of the blocks suffices for decoding. (Encoding will

\footnotetext{
${ }^{10} \mathrm{Or}$, more generally, from a subset $B$ of $\mathbb{Z}_{p}$ of appropriate size; see Section 1.1 .
} 
cause the file length to grow approximately $(1 / \rho) \times$.) Let $l$ be the number of indices in the query $Q$, and $B \subseteq \mathbb{Z}_{p}$ be the set from which the challenge weights $\nu_{i}$ are drawn.

Our proofs - see Section 4.2 for the details - guarantee that extraction will succeed from any adversary that convincingly answers an $\epsilon$-fraction of queries, provided that $\epsilon-\rho^{l}-1 / \# B$ is nonnegligible in $\lambda$. It is this requirement that guides the choice of parameters.

A conservative choice is $\rho=1 / 2, l=\lambda$, and $B=\{0,1\}^{\lambda}$; this guarantees extraction against any adversary 11 For applications that can tolerate a larger error rate these parameters can be reduced. For example, if a 1-in-1,000,000 error is acceptable, we can take $B$ to be the set of 22-bit strings and $l$ to be 22 ; alternatively, the coding expansion $1 / \rho$ can be reduced.

A tradeoff between storage and communication. As we have described our schemes above, each file block is accompanied by an authenticator of equal length. This gives a $2 \times$ overhead beyond that imposed by the erasure code, and the server's response in the proof-of-retrievability protocol is $2 \times$ the length of an authenticator. In the full schemes of Section 3 , we introduce a parameter $s$ that gives a tradeoff between storage overhead and response length. Each block consists of $s$ elements of $\mathbb{Z}_{p}$ that we call sectors. There is one authenticator per block, reducing the overhead to $(1+1 / s) \times$. The server's response is one aggregated block and authenticator, and is $(1+s) \times$ as long as an authenticator. The choice $s=1$ corresponds to our schemes as we described them above and to the scheme given by Ateniese et al. [3] ${ }^{12}$

Compressing the request. A request, as we have seen, consists of an $l$ element subset of $[1, n]$ together with $l$ elements of the coefficient set $B$, chosen uniformly and independently at random. In the conservative parametrization above, a request is thus $\lambda \cdot(\lceil\lg n\rceil+\lambda)$ bits long. One can reduce the randomness required to generate the request using standard techniques ${ }^{13}$ but this will not shorten the request itself. In the random oracle model, the verifier can send a short ( $2 \lambda$ bit) seed for the random oracle from which the prover will generate the full query. Using this technique we can make the queries as well as responses compact in our publicly verifiable scheme, which already relies on random oracles ${ }^{14}$ Obtaining short queries in the standard model is the major remaining open problem in proofs of retrievability.

We note that, by techniques similar to those discussed above, a PRF can be used to generate the per-file secret values $\left\{\alpha_{j}\right\}$ for our privately verifiable scheme and a random oracle seed can be used to generate the per-file public generators $\left\{u_{j}\right\}$ in our publicly verifiable scheme. This allows file tags for both schemes to be short: $O(\lambda)$, asymptotically.

We also note that subsequent to our work Bowers, Juels, and Oprea [9] provided a framework, based on "inner and outer" error correcting codes, by which they describe parameterizations of our approach that trade off the cost of a single audit and the computational efficiency of extracting a

\footnotetext{
${ }^{11}$ The careful analysis in our proofs allows us to show that, for 80-bit security, the challenge coefficients $\nu_{i}$ can be 80 bits long, not 160 as proposed in [4, p. 17]. The smaller these coefficients, the more efficient the multiplications or exponentiations that involve them.

${ }^{12}$ It would be possible to shorten the response further using knowledge-of-exponent assumptions, as Ateniese et al. do, but such assumptions are strong and nonstandard; more importantly, their use means that the extractor can never be implemented in the real world.

${ }^{13}$ For example, choose keys $k^{\prime}$ and $k^{\prime \prime}$ for PRFs with respective ranges $[1, n]$ and $B$. The query indices are the first $l$ distinct values amongst $f_{k^{\prime}}^{\prime}(1), f_{k^{\prime}}^{\prime}(2), \ldots$; the query coefficients are $f_{k^{\prime \prime}}^{\prime \prime}(1), \ldots, f_{k^{\prime \prime}}^{\prime \prime}(l)$.

14 Ateniese et al. propose to eliminate random oracles here by having the prover generate the full query using PRF keys sent by the verifier [4, p. 11], but it is not clear how to prove such a scheme secure, since the PRF security definition assumes that keys are kept secret.
} 
file a series of audit requests. In our work we have chosen to put emphasis on reducing single audit costs. We envision an audit as a mechanism to ensure that a file is indeed available and that a file under most circumstances will be retrieved as a simple bytestream.

\subsection{Our Proofs}

We provide a modular proof framework for the security of our schemes. Our framework allows us to argue about the systems unforgeability, extractability, and retrievability with these three parts based respectively on cryptographic, combinatorial, and coding-theoretical techniques. Only the first part differs between the three schemes we propose. The combinatorial techniques we develop are nontrivial and we believe they will be of independent interest.

It is interesting to compare both our security model and our proof methodology to those in related work.

The proof of retrievability model has two major distinctions from that used by Naor and Rothblum [25] (in addition to the public-key setting). First, the NR model assumes a checker can request and receive specific memory locations from the prover. In the proof of retrievability model, the prover can consist of an arbitrary program as opposed to a simple memory layout and this program may answer these questions in an arbitrary manner. We believe that this realistically represents an adversary in the type of setting we are considering. In the NR setting the extractor needs to retrieve the file given the server's memory; in the POR setting the analogy is that the extractor receives the adversary's program.

Second, in the proof of retrievability model we allow the attacker to execute a polynomial number of proof attempts before committing to how it will store memory. In the NR model the adversary does not get to execute the protocol before committing its memory. This weaker model is precisely what allows for the use of 1-bit MACs with error correcting codes in one NR variant. One might argue that in many situations this is sufficient. If a storage server responds incorrectly to an audit request we might assume that it is declared to be cheating and there is no need to go further. However, this limited view overlooks several scenarios. In particular, we want to be able to handle setups where there are several verifiers that do not communicate or if there might be several storage servers handling the same encoded file that are audited independently. Only our stronger model can correctly reflect these situations. In general, we believe that the strongest security model allows for a system to be secure in the most contexts including those not previously considered 15

One of the distinctive and challenging parts of our work is to argue extraction from homomorphically accumulated blocks. While Ateniese et al. [3] proposed using homomorphic RSA signatures and proved what is equivalent to our unforgeability requirement, they did not provide an argument that one could extract individual blocks from a prover. The only place where extractability is addressed in their work is a short paragraph in Appendix A, where they provide some intuitive arguments. Here is one concrete example: Their constructions make multiple uses of pseudorandom functions (PRFs), yet the security properties of a PRF are never applied in a security reduction. This gives compelling evidence that a rigorous security proof was not provided. Again, we emphasize that extraction is needed in even the weaker proof of data possession model claimed by the authors.

Extractability issues arise in several natural constructions. Proving extraction from aggregated

\footnotetext{
${ }^{15}$ We liken this argument to that for the strong definition currently accepted for chosen-ciphertext secure encryption.
} 
authenticator values can be challenging; in Appendix $\mathrm{B}$ we show an attack on a natural but incorrect system that is very similar to the "E-PDP" efficient alternative scheme given by Ateniese et al. (which they use in their performance measurements). For this scheme, Ateniese et al. claim only that the protocol establishes that a cheating prover has the sum $\sum_{i \in I} m_{i}$ of the blocks. We show that indeed this is all it can provide. Ateniese et al. calculate that a malicious server attacking the E-PDP scheme et al. that a malicious server attacking the E-PDP scheme would need to store $10^{140}$ blocks in order to cheat with probability $100 \%$. By contrast, our attack, which allows the server to cheat with somewhat lower probability (almost $9 \%$ for standard parameters) requires no more storage than were the server faithfully storing the file.

Finally, we argue that the POR is the "right" model for considering practical data storage problems, since provides a successful audit guarantees that all the data can be extracted. Other work has advocated that a weaker Proof of Data Possession [3] model might be acceptable. In this model, one only wants to guarantee that a certain percentage (e.g., 90\%) of data blocks are available. By offering this weaker guarantee one might hope to avoid the overhead of applying erasure codes. However, this weaker condition is unsatisfactory for most practical application demands. One might consider how happy a user would be were $10 \%$ of a file containing accounting data lost. Or if, for a compressed file, the compression tables were lost - and with them all useful data. Instead of hoping that there is enough redundancy left to reconstruct important data in an ad-hoc way, it is much more desirable to have a model that inherently provides this. We also note that Ateniese et al. [3] make an even weaker guarantee for their "E-PDP" system that they implement and use as the basis for their measurements. According to [3] their E-PDP system "only guarantees possession of the sum of the blocks." While this might be technically correct, it is even more difficult to discern what direct use could come from retrieving a sum of a subset of data blocks.

One might still hope to make use of systems proved secure under these models. For example, we might attempt to make a PDP system usable by adding on an erasure encoding step. In addition, if a system proved that one could be guaranteed sums of blocks for a particular audit, then it might be the case that by using multiple audit one could guarantee that individual file blocks could be extracted. However, one must prove that this is the case and account for the additional computational and communication overhead of multiple passes. When systems use definitions that don't model full retrievability it becomes very difficult to make any useful security or performance comparisons.

\section{Security Model}

We recall the security definition of Juels and Kaliski [20]. Our version differs from the original definition in several details:

- we rule out any state (" $\alpha$ ") in key generation and in verification, because (as explained in Section 1) we believe that verifiers in proof-of-retrievability schemes should be stateless;

- we allow the proof protocol to be arbitrary, rather than two-move, challenge-response; and

- our key generation emits a public key as well as a private key, to allow us to capture the notion of public verifiability. 
Note that any stateless scheme secure in the original Juels-Kaliski model will be secure in our variant, and any scheme secure in our variant whose proof protocol can be cast as two-move, challenge-response protocol will be secure in the Juels-Kaliski definition. In particular, our scheme with private verifiability is secure in the original Juels-Kaliski model 16

A proof of retrievability scheme defines four algorithms, $\mathrm{Kg}, \mathrm{St}, \mathcal{V}$, and $\mathcal{P}$, which behave thus:

$\mathbf{K g}()$. This randomized algorithm generates a public-private keypair ( $p k, s k)$.

St $(s k, M)$. This randomized file-storing algorithm takes a secret key sk and a file $M \in\{0,1\}^{*}$ to store. It processes $M$ to produce and output $M^{*}$, which will be stored on the server, and a tag $t$. The tag contains information that names the file being stored; it could also contain additional secret information encrypted under the secret key sk.

$\mathcal{P}, \mathcal{V}$. The randomized proving and verifying algorithms define a protocol for proving file retrievability. During protocol execution, both algorithms take as input the public key pk and the file tag $t$ output by St. The prover algorithm also takes as input the processed file description $M^{*}$ that is output by St, and the verifier algorithm takes as input the secret key. At the end of the protocol run, $\mathcal{V}$ outputs 0 or 1 , where 1 means that the file is being stored on the server. We can denote a run of two machines executing the algorithms as: $\{0,1\} \stackrel{\mathrm{R}}{\leftarrow}\left(\mathcal{V}(p k, s k, t) \rightleftharpoons \mathcal{P}\left(p k, t, M^{*}\right)\right)$.

We would like a proof-of-retrievability protocol to be correct and sound. Correctness requires that, for all keypairs $(p k, s k)$ output by $\mathrm{Kg}$, for all files $M \in\{0,1\}^{*}$, and for all $\left(M^{*}, t\right)$ output by $\operatorname{St}(s k, M)$, the verification algorithm accepts when interacting with the valid prover:

$$
\left(\mathcal{V}(p k, s k, t) \rightleftharpoons \mathcal{P}\left(p k, t, M^{*}\right)\right)=1 .
$$

A proof-of-retrievability protocol is sound if any cheating prover that convinces the verification algorithm that it is storing a file $M$ is actually storing that file, which we define in saying that it yields up the file $M$ to an extractor algorithm that interacts with it using the proof-of-retrievability protocol. We formalize the notion of an extractor and then give a precise definition for soundness.

An extractor algorithm $\operatorname{Extr}\left(p k, s k, t, \mathcal{P}^{\prime}\right)$ takes the public and private keys, the file tag $t$, and the description of a machine implementing the prover's role in the proof-of-retrievability protocol: for example, the description of an interactive Turing machine, or of a circuit in an appropriately augmented model. The algorithm's output is the file $M \in\{0,1\}^{*}$. Note that Extr is given nonblack-box access to $\mathcal{P}^{\prime}$ and can, in particular, rewind it.

Consider the following setup game between an adversary $\mathcal{A}$ and an environment:

1. The environment generates a keypair ( $p k, s k)$ by running $\mathrm{Kg}$, and provides $p k$ to $\mathcal{A}$.

2. The adversary can now interact with the environment. It can make queries to a store oracle, providing, for each query, some file $M$. The environment computes $\left(M^{*}, t\right) \stackrel{\mathrm{R}}{\leftarrow} \mathrm{St}(s k, M)$ and returns both $M^{*}$ and $t$ to the adversary.

\footnotetext{
${ }^{16}$ In an additional minor difference, we do not specify the extraction algorithm as part of a scheme, because we do not expect that the extract algorithm will be deployed in outsourced storage applications. Nevertheless, the extract algorithm used in our proofs (cf. Section 4.2) is quite simple: undertake many random $\mathcal{V}$ interactions with the cheating prover; keep track of those queries for which $\mathcal{V}$ accepts the cheating prover's reply as valid; and continue until enough information has been gathered to recover file blocks by means of linear algebra. The adversary $\mathcal{A}$ could implement this algorithm by means of its proof-of-retrievability protocol access.
} 
3. For any $M$ on which it previously made a store query, the adversary can undertake executions of the proof-of-retrievability protocol, by specifying the corresponding tag $t$. In these protocol executions, the environment plays the part of the verifier and the adversary plays the part of the prover: $\mathcal{V}(p k, s k, t) \rightleftharpoons \mathcal{A}$. When a protocol execution completes, the adversary is provided with the output of $\mathcal{V}$. These protocol executions can be arbitrarily interleaved with each other and with the store queries described above.

4. Finally, the adversary outputs a challenge tag $t$ returned from some store query, and the description of a prover $\mathcal{P}^{\prime}$.

The cheating prover $\mathcal{P}^{\prime}$ is $\epsilon$-admissible if it convincingly answers an $\epsilon$ fraction of verification challenges, i.e., if $\operatorname{Pr}\left[\left(\mathcal{V}(p k, s k, t) \rightleftharpoons \mathcal{P}^{\prime}\right)=1\right] \geq \epsilon$. Here the probability is over the coins of the verifier and the prover. Let $M$ be the message input to the store query that returned the challenge tag $t$ (along with a processed version $M^{*}$ of $M$ ).

Definition 2.1. We say a proof-of-retrievability scheme is $\epsilon$-sound if there exists an extraction algorithm Extr such that, for every adversary $\mathcal{A}$, whenever $\mathcal{A}$, playing the setup game, outputs an $\epsilon$-admissible cheating prover $\mathcal{P}^{\prime}$ for a file $M$, the extraction algorithm recovers $M$ from $\mathcal{P}^{\prime}$-i.e., $\operatorname{Extr}\left(p k, s k, t, \mathcal{P}^{\prime}\right)=M$ - except possibly with negligible probability.

Note that it is okay for $\mathcal{A}$ to have engaged in the proof-of-retrievability protocol for $M$ in its interaction with the environment. Note also that each run of the proof-of-retrievability protocol is independent: the verifier implemented by the environment is stateless.

Finally, note that we require that extraction succeed (with all but negligible probability) from an adversary that causes $\mathcal{V}$ to accept with any nonnegligible probability $\epsilon$. An adversary that passes the verification even a very small but nonnegligible fraction of the time - say, once in a million interactions - is fair game. Intuitively, recovering enough blocks to reconstruct the original file from such an adversary should take $O(n / \epsilon)$ interactions; our proofs achieve essentially this bound.

Concrete or asymptotic formalization. A proof-of-retrievability scheme is secure if no efficient algorithm wins the game above except rarely, where the precise meaning of "efficient" and "rarely" depends on whether we employ a concrete of asymptotic formalization.

It is possible to formalize the notation above either concretely or asymptotically. In a concrete formalization, we require that each algorithm defining the proof-of-retrievability scheme run in at most some number of steps, and that for any algorithm $\mathcal{A}$ that runs in time $t$ steps, that makes at most $q_{S}$ store queries, and that undertakes at most $q_{P}$ proof-of-retrievability protocol executions, extraction from an $\epsilon$-admissible prover succeeds except with some small probability $\delta$. In an asymptotic formalization, every algorithm is provided with an additional parameter $1^{\lambda}$ for security parameter $\lambda$, we require each algorithm to run in time polynomial in $\lambda$, and we require that extraction fail from an $\epsilon$-admissible prover with only negligible probability in $\lambda$, provided $\epsilon$ is nonnegligible.

Public or private verification, public or private extraction. In the model above, the verifier and extractor are provided with a secret that is not known to the prover or other parties. This is a secret-verification, secret-extraction model model. If the verification algorithm does not use the secret key, any third party can check that a file is being stored, giving public verification. Similarly, if the extract algorithm does not use the secret key, any third party can extract the file from a server, giving public extraction. 


\section{Constructions}

In this section we give formal descriptions for both our private and public verification systems. The systems here follow the constructions outlined in the introduction with a few added generalizations. First, we allow blocks to contain $s \geq 1$ elements of $\mathbb{Z}_{p}$. This allows for a tradeoff between storage overhead and communication overhead. Roughly the communication complexity grows as $s+1$ elements of $\mathbb{Z}_{p}$ and the ratio of authentication overhead to data stored (post encoding) is $1: s$. Second, we describe our systems where the set of coefficients sampled from $B$ can be smaller than all of $\mathbb{Z}_{p}$. This enables us to take advantage make more efficient systems in certain situations.

\subsection{Common Notation}

We will work in the group $\mathbb{Z}_{p}$. When we work in the bilinear setting, the group $\mathbb{Z}_{p}$ is the support of the bilinear group $G$, i.e., $\# G=p$. In queries, coefficients will come from a set $B \subseteq \mathbb{Z}_{p}$. For example, $B$ could equal $\mathbb{Z}_{p}$, in which case query coefficients will be randomly chosen out of all of $\mathbb{Z}_{p}$.

After a file undergoes preliminary processing, the processed file is split into blocks, and each block is split into sectors. Each sector is one element of $\mathbb{Z}_{p}$, and there are $s$ sectors per block. If the processed file is $b$ bits long, then there are $n=\lceil b / s \lg p\rceil$ blocks. We will refer to individual file sectors as $\left\{m_{i j}\right\}$, with $1 \leq i \leq n$ and $1 \leq j \leq s$.

Queries. A query is an $l$-element set $Q=\left\{\left(i, \nu_{i}\right)\right\}$. Each entry $\left(i, \nu_{i}\right) \in Q$ is such that $i$ is a block index in the range $[1, n]$, and $\nu_{i}$ is a multiplier in $B$. The size $l$ of $Q$ is a system parameter, as is the choice of the set $B$.

The verifier chooses a random query as follows. First, she chooses, uniformly at random, an $l$-element subset $I$ of $[1, n]$. Then, for each element $i \in I$ she chooses, uniformly at random, an element $\nu_{i} \stackrel{\mathrm{R}}{\leftarrow} B$. We observe that this procedure implies selection of $l$ elements from $[1, n]$ without replacement but a selection of $l$ elements from $B$ with replacement.

Although the set notation $Q=\left\{\left(i, \nu_{i}\right)\right\}$ is space-efficient and convenient for implementation, we will also make use of a vector notation in the analysis. A query $Q$ over indices $I \subset[1, n]$ is represented by a vector $\mathbf{q} \in\left(\mathbb{Z}_{p}\right)^{n}$ where $\mathbf{q}_{i}=\nu_{i}$ for $i \in I$ and $\mathbf{q}_{i}=0$ for all $i \notin I$. Equivalently, letting $\mathbf{u}_{1}, \ldots, \mathbf{u}_{n}$ be the usual basis for $\left(\mathbb{Z}_{p}\right)^{n}$, we have $\mathbf{q}=\sum_{\left(i, \nu_{i}\right) \in Q} \nu_{i} \mathbf{u}_{i} \cdot{ }^{17}$.

If the set $B$ does not contain 0 then a random query (according to the selection procedure defined above) is a random weight- $l$ vector in $\left(\mathbb{Z}_{p}\right)^{n}$ with coefficients in $B$. If $B$ does contain 0 , then a similar argument can be made, but care must be taken to distinguish the case " $i \in I$ and $\nu_{i}=0$ " from the case " $i \notin I$."

Aggregation. For its response, the server responds to a query $Q$ by computing, for each $j$, $1 \leq j \leq s$, the value

$$
\mu_{j} \leftarrow \sum_{\left(i, \nu_{i}\right) \in Q} \nu_{i} m_{i j}
$$

That is, by combining sectorwise the blocks named in $Q$, each with its multiplier $\nu_{i}$. Addition, of course, is modulo $p$. The response is $\left(\mu_{1}, \ldots, \mu_{s}\right) \in\left(\mathbb{Z}_{p}\right)^{s}$.

\footnotetext{
${ }^{17}$ We are using subscripts to denote vector elements (for $\mathbf{q}$ ) and to choose a particular vector from a set (for $\mathbf{u}$ ); but no confusion should arise.
} 
Suppose we view the message blocks on the server as an $n \times s$ element matrix $M=\left(m_{i j}\right)$, then, using the vector notation for queries given above, the server's response is given by $\mathbf{q} M$.

\subsection{Construction for Private Verification}

Let $f:\{0,1\}^{*} \times \mathcal{K}_{\text {prf }} \rightarrow \mathbb{Z}_{p}$ be a PRF ${ }^{18}$ The construction of the private verification scheme Priv is:

Priv. $\mathrm{Kg}()$. Choose a random symmetric encryption key $k_{\text {enc }} \stackrel{R}{\leftarrow} \mathcal{K}_{\text {enc }}$ and a random MAC key $k_{\text {mac }} \stackrel{R}{\leftarrow} \mathcal{K}_{\text {mac }}$. The secret key is $s k=\left(k_{\text {enc }}, k_{\text {mac }}\right)$; there is no public key.

Priv.St $(s k, M)$. Given the file $M$, first apply the erasure code to obtain $M^{\prime}$; then split $M^{\prime}$ into $n$ blocks (for some $n$ ), each $s$ sectors long: $\left\{m_{i j}\right\}_{\substack{1 \leq i \leq n \\ 1 \leq j \leq s}}$. Now choose a PRF key $k_{\text {prf }} \stackrel{R}{\leftarrow} \mathcal{K}_{\text {prf }}$ and $s$ random numbers $\alpha_{1}, \ldots, \alpha_{s} \stackrel{\mathrm{R}}{\leftarrow} \mathbb{Z}_{p}$. Let $t_{0}$ be $n \|$ Enc $_{k_{\text {enc }}}\left(k_{\text {prf }}\left\|\alpha_{1}\right\| \cdots \| \alpha_{s}\right)$; the file tag is $t=t_{0} \| \mathrm{MAC}_{k_{\text {mac }}}\left(t_{0}\right)$. Now, for each $i, 1 \leq i \leq n$, compute

$$
\sigma_{i} \leftarrow f_{k_{\mathrm{prf}}}(i)+\sum_{j=1}^{s} \alpha_{j} m_{i j}
$$

The processed file $M^{*}$ is $\left\{m_{i j}\right\}, 1 \leq i \leq n, 1 \leq j \leq s$ together with $\left\{\sigma_{i}\right\}, 1 \leq i \leq n$.

Priv. $\mathcal{V}(p k, s k, t)$. Parse $s k$ as $\left(k_{\mathrm{enc}}, k_{\mathrm{mac}}\right)$. Use $k_{\mathrm{mac}}$ to verify the MAC on $t$; if the MAC is invalid, reject by emitting 0 and halting. Otherwise, parse $t$ and use $k_{\text {enc }}$ to decrypt the encrypted portions, recovering $n, k_{\text {prf }}$, and $\alpha_{1}, \ldots, \alpha_{s}$. Now pick a random $l$-element subset $I$ of the set $[1, n]$, and, for each $i \in I$, a random element $\nu_{i} \stackrel{R}{\leftarrow} B$. Let $Q$ be the set $\left\{\left(i, \nu_{i}\right)\right\}$. Send $Q$ to the prover.

Parse the prover's response to obtain $\mu_{1}, \ldots, \mu_{s}$ and $\sigma$, all in $\mathbb{Z}_{p}$. If parsing fails, fail by emitting 0 and halting. Otherwise, check whether

$$
\sigma \stackrel{?}{=} \sum_{\left(i, \nu_{i}\right) \in Q} \nu_{i} f_{k_{\mathrm{prf}}}(i)+\sum_{j=1}^{s} \alpha_{j} \mu_{j}
$$

if so, output 1 ; otherwise, output 0 .

Priv. $\mathcal{P}\left(p k, t, M^{*}\right)$. Parse the processed file $M^{*}$ as $\left\{m_{i j}\right\}, 1 \leq i \leq n, 1 \leq j \leq s$, along with $\left\{\sigma_{i}\right\}$, $1 \leq i \leq n$. Parse the message sent by the verifier as $Q$, an $l$-element set $\left\{\left(i, \nu_{i}\right)\right\}$, with the $i$ 's distinct, each $i \in[1, n]$, and each $\nu_{i} \in B$. Compute

$$
\mu_{j} \leftarrow \sum_{\left(i, \nu_{i}\right) \in Q} \nu_{i} m_{i j} \quad \text { for } 1 \leq j \leq s, \quad \text { and } \quad \sigma \leftarrow \sum_{\left(i, \nu_{i}\right) \in Q} \nu_{i} \sigma_{i} .
$$

Send to the prover in response the values $\mu_{1}, \ldots, \mu_{s}$ and $\sigma$.

\footnotetext{
${ }^{18}$ In fact, the domain need only be $\lceil\lg N\rceil$-bit strings, where $N$ is a bound on the number of blocks in a file.
} 


\subsection{Construction for Public Verification}

Let $e: G \times G \rightarrow G_{T}$ be a bilinear map, let $g$ be a generator of $G$, and let $H:\{0,1\}^{*} \rightarrow G$ be the BLS hash, treated as a random oracle 19 The construction of the public verification scheme Pub is:

Pub.Kg(). Generate a random signing keypair (spk, ssk) $\stackrel{R}{\leftarrow} \mathrm{SKg}$. Choose a random $\alpha \stackrel{R}{\leftarrow} \mathbb{Z}_{p}$ and compute $v \leftarrow g^{\alpha}$. The secret key is $s k=(\alpha, s s k)$; the public key is $p k=(v, s p k)$.

Pub.St $(s k, M)$. Given the file $M$, first apply the erasure code to obtain $M^{\prime}$; then split $M^{\prime}$ into $n$ blocks (for some $n$ ), each $s$ sectors long: $\left\{m_{i j}\right\}_{\substack{1 \leq i \leq n \\ 1 \leq j \leq s}}$. Now parse sk as $(\alpha, s s k)$. Choose a random file name name from some sufficiently large domain (e.g., $\mathbb{Z}_{p}$ ). Choose $s$ random elements $u_{1}, \ldots, u_{s} \stackrel{\mathrm{R}}{\leftarrow} G$. Let $t_{0}$ be "name $\|n\| u_{1}\|\cdots\| u_{s}$ "; the file tag $t$ is $t_{0}$ together with a signature on $t_{0}$ under private key ssk: $t \leftarrow t_{0} \| \operatorname{SSig}_{\text {ssk }}\left(t_{0}\right)$. For each $i, 1 \leq i \leq n$, compute

$$
\sigma_{i} \leftarrow\left(H(\text { name } \| i) \cdot \prod_{j=1}^{s} u_{j}^{m_{i j}}\right)^{\alpha}
$$

The processed file $M^{*}$ is $\left\{m_{i j}\right\}, 1 \leq i \leq n, 1 \leq j \leq s$ together with $\left\{\sigma_{i}\right\}, 1 \leq i \leq n$.

Pub. $\mathcal{V}(p k, s k, t)$. Parse $p k$ as $(v, s p k)$. Use spk to verify the signature on on $t$; if the signature is invalid, reject by emitting 0 and halting. Otherwise, parse $t$, recovering name, $n$, and $u_{1}, \ldots, u_{s}$. Now pick a random $l$-element subset $I$ of the set $[1, n]$, and, for each $i \in I$, a random element $\nu_{i} \stackrel{\mathrm{R}}{\leftarrow} B$. Let $Q$ be the set $\left\{\left(i, \nu_{i}\right)\right\}$. Send $Q$ to the prover.

Parse the prover's response to obtain $\left(\mu_{1}, \ldots, \mu_{s}\right) \in\left(\mathbb{Z}_{p}\right)^{s}$ and $\sigma \in G$. If parsing fails, fail by emitting 0 and halting. Otherwise, check whether

$$
e(\sigma, g) \stackrel{?}{=} e\left(\prod_{\left(i, \nu_{i}\right) \in Q} H(\text { name } \| i)^{\nu_{i}} \cdot \prod_{j=1}^{s} u_{j}^{\mu_{j}}, v\right)
$$

if so, output 1; otherwise, output 0 .

Pub.P $\left(p k, t, M^{*}\right)$. Parse the processed file $M^{*}$ as $\left\{m_{i j}\right\}, 1 \leq i \leq n, 1 \leq j \leq s$, along with $\left\{\sigma_{i}\right\}$, $1 \leq i \leq n$. Parse the message sent by the verifier as $Q$, an $l$-element set $\left\{\left(i, \nu_{i}\right)\right\}$, with the $i$ 's distinct, each $i \in[1, n]$, and each $\nu_{i} \in B$. Compute

$$
\mu_{j} \leftarrow \sum_{\left(i, \nu_{i}\right) \in Q} \nu_{i} m_{i j} \in \mathbb{Z}_{p} \quad \text { for } 1 \leq j \leq s, \quad \text { and } \quad \sigma \leftarrow \prod_{\left(i, \nu_{i}\right) \in Q} \sigma_{i}^{\nu_{i}} \in G
$$

Send to the prover in response the values $\mu_{1}, \ldots, \mu_{s}$ and $\sigma$.

\footnotetext{
${ }^{19}$ For notational simplicity, we present our scheme using a symmetric bilinear map, but efficient implementations will use an asymmetric map $e: G_{1} \times G_{2} \rightarrow G_{T}$. Translating our scheme to this setting is simple. User public keys $v$ will live in $G_{2}$; file generators $u_{j}$ will live in $G_{1}$, as will the output of $H$; and security will be reduced to co-CDH [8].
} 


\section{Security Proofs}

In this section we prove that both of our systems are secure under the model we provided. Intutively, we break our proof into three parts. The first part shows that the attacker can never give a forged response back to the a verifier. The second part of the proof shows that from any adversary that passes the check a non-negligible amount of the time we will be able to extract a constant fraction of the encoded blocks. The second step uses the fact that (w.h.p.) all verified responses must be legitimate. Finally, we show that if this constant fraction of blocks is recovered we can use the erasure code to reconstruct the original file.

The proof, for both schemes, is in three parts:

1. Prove that the verification algorithm will reject except when the prover's $\left\{\mu_{j}\right\}$ are correctly computed, i.e., are such that $\mu_{j}=\sum_{\left(i, \nu_{i}\right) \in Q} \nu_{i} m_{i j}$. This part of the proof uses cryptographic techniques.

2. Prove that the extraction procedure can efficiently reconstruct a $\rho$ fraction of the file blocks when interacting with a prover that provides correctly-computed $\left\{\mu_{j}\right\}$ responses for a nonnegligible fraction of the query space. This part of the proof uses combinatorial techniques.

3. Prove that a $\rho$ fraction of the blocks of the erasure-coded file suffice for reconstructing the original file. This part of the proof uses coding theory techniques.

The crucial point is the second and third parts of the proof are identical for our two schemes; only the first part is different.

\subsection{Part-One Proofs}

\subsubsection{Scheme with Private Verifiability}

Theorem 4.1. If the MAC scheme is unforgeable, the symmetric encryption scheme is semantically secure, and the PRF is secure, then (except with negligible probability) no adversary against the soundness of our private-verification scheme ever causes $\mathcal{V}$ to accept in a proof-of-retrievability protocol instance, except by responding with values $\left\{\mu_{j}\right\}$ and $\sigma$ that are computed correctly, i.e., as they would be by Priv.P.

We prove the theorem in a series of games.

Game 0. The first game, Game 0, is simply the challenge game defined in Section 2 .

Game 1. Game 1 is the same as Game 0, with one difference. The challenger keeps a list of all MAC-authenticated tags ever issued as part of a store-protocol query. If the adversary ever submits a tag $t$ either in initiating a proof-of-storage protocol or as the challenge tag, that (1) verifies as valid under $k_{\text {mac }}$ but (2) is not a tag authenticated by the challenger, the challenger declares failure and aborts.

Clearly, if there is a difference in the adversary's success probability between Games 0 and 1, we can use the adversary to construct a forger against the MAC scheme. 
Game 2. In Game 2, the challenger includes in the tags not the encryption of $k_{\text {prf }}\left\|\alpha_{1}\right\| \cdots \| \alpha_{s}$ but a random bit-string of the same length. When given a tag by the adversary, the challenger uses the values that would (in previous games) have been encrypted in the tag.

Note that the modification made in Game 1 ensures that the challenger never sees a tag except those it issued through St queries, so it need never actually decrypt a tag portion.

Clearly, if there is a difference in the adversary's success probability between Games 1 and 2, we can use the adversary to break the semantic security of the symmetric encryption scheme. Note that the reduction so obtained will suffer a $1 / q_{S}$ security loss, where $q_{S}$ is the number of St queries made by the adversary, because we must use a hybrid argument between "all valid encryptions" and "no valid encryptions."

Game 3. In Game 3, the challenger picks uses truly random values in $\mathbb{Z}_{p}$ instead of PRF output, remembering these values to use when verifying the adversary's responses in proof-of-storage protocol instances. More specifically, the challenger evaluates $f_{k_{\mathrm{prf}}}(i)$ not by applying the PRF algorithm but by generating a random value $r \stackrel{R}{\leftarrow} \mathbb{Z}_{p}$ and inserting an entry $\left(k_{\text {prf }}, i, r\right)$ in a table; it consults this table when evaluating the PRF to ensure consistency.

If there is a difference in the adversary's success probability between Games 2 and 3, we can use the adversary to break the security of the PRF. As before, a hybrid argument necessitates a security loss in the reduction; this time, the loss is $1 /\left(N q_{S}\right)$, where $N$ is a bound on the number of blocks in the encoding of any file the adversary requests to have stored.

Game 4. In Game 4, the challenger keeps a table of its responses to St queries made by the adversary. It observes each instance of the proof-of-storage protocol with the adversary - whether because of a proof-of-storage query made by the adversary, or in the test made of $\mathcal{P}^{\prime}$, or as part of the extraction attempt by Extr. If in any of these interactions the adversary responds with in a way that (1) passes the verification algorithm but (2) is not what would have been computed by an honest prover, the challenger declares failure and aborts.

More specifically, suppose the protocol instance involves an $n$-block file with secret values $\alpha_{1}, \ldots, \alpha_{s}$, contains sectors $\left\{m_{i j}\right\}$, and the block signatures issued by St are $\left\{\sigma_{i}\right\}$. Suppose $Q=\left\{\left(i, \nu_{i}\right)\right\}$ is the query that causes the challenger to abort, and that the adversary's response to that query was $\mu_{1}^{\prime}, \ldots, \mu_{s}^{\prime}$ together with $\sigma^{\prime}$. Let the expected response - i.e., the one that would have been obtained from an honest prover - be $\mu_{1}, \ldots, \mu_{s}$ and $\sigma$, where $\sigma=\sum_{\left(i, \nu_{i}\right) \in Q} \nu_{i} \sigma_{i}$ and $\mu_{j}=\sum_{\left(i, \nu_{i}\right) \in Q} \nu_{i} m_{i j}$ for $1 \leq j \leq s$. If the adversary's response satisfies the verifier - i.e., if $\sigma^{\prime}=\sum_{\left(i, \nu_{i}\right) \in Q} \nu_{i} r_{k_{\mathrm{pr} f}, i}+\sum_{j=1}^{s} \alpha_{j} \mu_{j}^{\prime}$, where $r_{k_{\mathrm{pr} f}, i}$ is the random value substituted by Game 2 for $f_{k_{\text {prf }}}(i)$, but $\mu_{j}^{\prime} \neq \mu_{j}$ for at least one $j$, the challenger aborts. (If $\mu_{j}^{\prime}=\mu_{j}$ for all $j$ but $\sigma^{\prime} \neq \sigma$, it is impossible that the verification equation holds, so we need not worry about this case.)

By the correctness of the scheme the expected values $\sigma$ along with $\left\{\mu_{j}\right\}$ also satisfy the verification equation, so we have $\sigma=\sum_{i \in I} r_{k_{\mathrm{pr}}, i}+\sum_{j=1}^{s} \alpha_{j} \mu_{j}$. Letting $\Delta \sigma \stackrel{\text { def }}{=} \sigma^{\prime}-\sigma$ and $\Delta \mu_{j} \stackrel{\text { def }}{=} \mu_{j}^{\prime}-\mu_{j}$ for $1 \leq j \leq s$ and subtracting the verification equation for $\sigma$ from that for $\sigma^{\prime}$, we have

$$
\Delta \sigma=\sum_{j=1}^{s} \alpha_{j} \Delta \mu_{j}
$$

We can think of $\left\{\Delta \mu_{j}\right\}$ and $\Delta \sigma$ as variables in an $(s+1)$-dimensional space. The values for these variables that are accepted by the verifier are exactly those that satisfy equation (1), i.e., 
those that lie on a certain $s$-dimensional hyperplane. Any choice of values $\left\{\Delta \mu_{j}\right\}$ and $\Delta \sigma$ on this hyperplane, aside from the all-zero assignment that corresponds to the honest prover's answer, will cause the challenger to abort.

We will proceed by induction on the adversary's proof-of-storage protocol instances for any file, proving that if the adversary had not caused an abort in its first $i$ protocol instances, it will not cause an abort in its $(i+1)$ st protocol instance except with negligible probability.

At the time of the adversary's first protocol execution, the values $\alpha_{1}, \ldots, \alpha_{s}$ are independent of its view: They are no longer encrypted in the tag, and their only other appearance is in computing $\sigma_{i}=r_{k_{\mathrm{pr},}, i}+\sum_{j=1}^{s} \alpha_{j} m_{i j}$ for $1 \leq i \leq n$; but the random value $r_{k_{\mathrm{pr}}, i}$ replacing $f_{k_{\mathrm{prf}}}(i)$ (and used only there) means that $\sigma_{i}$ is independent of $\alpha_{1}, \ldots, \alpha_{j}$. Thus the probability that the values $\left\{\Delta \mu_{j}\right\}$ and $\Delta \sigma$ it chooses lie on the abort hyperplane is $1 / p$, so execution continues with probability $1-1 / p$.

The adversary learns some information about the abort-hyperplane from the rejection of its response: he learns that the point he queried is not on the hyperplane, and neither is any point on the line between that point and the origin. For his second protocol execution, he can choose a point on a plane containing this line but not on the line; this point lies on the abort-hyperplane with probability $1 /(p-1)$. No other way of choosing a point allows him a success probabilty so high.

More generally, after $i$ executions, the adversary has eliminated $i$ lines between his each of his $i$ points and the origin. Thus for his $(i+1)$ st execution he can succeed in naming a point on the abort-hyperplane with probability at most $1 /(p-i)$. To achieve this bound, he must choose all his points from a single plane passing through the origin; with this strategy, each rejection allows him to eliminate $p$ points out of the $p^{2}$ points on the plane.

To complete the induction, we see that the challenger aborts at some point during the adversary's $q$ protocol executions with probability

$$
1-\left(1-\frac{1}{p}\right)\left(1-\frac{1}{p-1}\right) \cdots\left(1-\frac{1}{p-q+1}\right)
$$

which is negligible. (This argument is inspired by Cramer and Shoup's analysis of their encryption scheme [14].)

Wrapping up. In Game 4, the adversary is constrained from answering any verification query with values other than those that would have been computed by Priv.P. Yet we have argued that, assuming the MAC, encryption scheme, and PRF are secure, there is only a negligible difference in the success probability of the adversary in this game compared to Game 1, where the adversary is not constrained in this manner. This completes the proof of Theorem 4.1 .

\subsubsection{Scheme with Public Verifiability}

Theorem 4.2. If the signature scheme used for file tags is existentially unforgeable and the computational Diffie-Hellman problem is hard in bilinear groups, then, in the random oracle model, except with negligible probability no adversary against the soundness of our public-verification scheme ever ever causes $\mathcal{V}$ to accept in a proof-of-retrievability protocol instance, except by responding with values $\left\{\mu_{j}\right\}$ and $\sigma$ that are computed correctly, i.e., as they would be by Pub.P.

Once again, we prove the theorem as a series of games. 
Game 0. The first game, Game 0, is simply the challenge game defined in Section 2, with the changes for public verifiability sketched at the end of that section.

Game 1. Game 1 is the same as Game 0, with one difference. The challenger keeps a list of all signed tags ever issued as part of a store-protocol query. If the adversary ever submits a tag $t$ either in initiating a proof-of-retrievability protocol or as the challenge tag, that (1) has a valid signature under ssk but (2) is not a tag signed by the challenger, the challenger declares failure and aborts.

Clearly, if there is a difference in the adversary's success probability between Games 0 and 1, we can use the adversary to construct a forger against the signature scheme.

Game 2. Game 2 is the same as Game 1, with one difference. The challenger keeps a list of its responses to St queries made by the adversary. Now the challenger observes each instance of the proof-of-retrievability protocol with the adversary - whether because of a proof-of-retrievability query made by the adversary, or in the test made of $\mathcal{P}^{\prime}$, or as part of the extraction attempt by Extr. If in any of these instances the adversary is successful (i.e., $\mathcal{V}$ outputs 1 ) but the adversary's aggregate signature $\sigma$ is not equal to $\prod_{\left(i, \nu_{i}\right) \in Q} \sigma_{i}^{\nu_{i}}$ (where $Q$ is the challenge issued by the verifier and $\sigma_{i}$ are the signatures on the blocks of the file considered in the protocol instance) the challenger declares failure and aborts.

Before analyzing the difference in success probabilities between Games 1 and 2, we will establish some notation and draw a few conclusions. Suppose the file that causes the abort is $n$ blocks long, has name name, has generating exponents $\left\{u_{j}\right\}$, and contains sectors $\left\{m_{i j}\right\}$, and that the block signatures issued by St are $\left\{\sigma_{i}\right\}$. Suppose $Q=\left\{\left(i, \nu_{i}\right)\right\}$ is the query that causes the challenger to abort, and that the adversary's response to that query was $\mu_{1}^{\prime}, \ldots, \mu_{s}^{\prime}$ together with $\sigma^{\prime}$. Let the expected response - i.e., the one that would have been obtained from an honest prover - be $\mu_{1}, \ldots, \mu_{s}$ and $\sigma$, where $\sigma=\prod_{\left(i, \nu_{i}\right) \in Q} \sigma_{i}^{\nu_{i}}$ and $\mu_{j}=\sum_{\left(i, \nu_{i}\right) \in Q} \nu_{i} m_{i j}$ for $1 \leq j \leq s$. By the correctness of the scheme, we know that the expected response satisfies the verification equation, i.e., that

$$
e(\sigma, g)=e\left(\prod_{\left(i, \nu_{i}\right) \in Q} H(\text { name } \| i)^{\nu_{i}} \cdot \prod_{j=1}^{s} u_{j}^{\mu_{j}}, v\right) ;
$$

Because the challenger aborted, we know that $\sigma \neq \sigma^{\prime}$ and that $\sigma^{\prime}$ passes the verification equation, i.e., that

$$
e\left(\sigma^{\prime}, g\right)=e\left(\prod_{\left(i, \nu_{i}\right) \in Q} H(\text { name } \| i)^{\nu_{i}} \cdot \prod_{j=1}^{s} u_{j}^{\mu_{j}^{\prime}}, v\right),
$$

where $v=g^{\alpha}$ is part of the challenger's public key. Observe that if $\mu_{j}^{\prime}=\mu_{j}$ for each $j$, it follows from the verification equation that $\sigma^{\prime}=\sigma$, which contradicts our assumption above. Therefore, if we define $\Delta \mu_{j} \stackrel{\text { def }}{=} \mu_{j}^{\prime}-\mu_{j}$ for $1 \leq j \leq s$, it must be the case that at least one of $\left\{\Delta \mu_{j}\right\}$ is nonzero.

With this in mind, we now show that if there is a nonnegligible difference in the adversary's success probabilities between Games 1 and 2 we can construct a simulator that solves the computational Diffie-Hellman problem.

The simulator is given as inputs values $g, g^{\alpha}, h \in G$; its goal is to output $h^{\alpha}$. The simulator behaves like the Game 1 challenger, with the following differences:

- In generating a key, it sets the public key $v$ to $g^{\alpha}$ received in the challenge. This means that it does not know the corresponding secret key $\alpha$. 
- The simulator programs the random oracle $H$. It keeps a list of queries and responses to answers consistently. In answering the adversary's queries it chooses a random $r \stackrel{\mathrm{R}}{\leftarrow} \mathbb{Z}_{p}$ and responds with $g^{r} \in G$. It also answers queries of the form $H($ name $\| i)$ in a special way, as we will see below.

- When asked to store some file whose coded representation comprises the $n$ blocks $\left\{m_{i j}\right\}$, $1 \leq i \leq n, 1 \leq j \leq s$, the simulator behaves as follows. It chooses a name name at random. Because the space from which names are drawn is large, it follows that, except with negligible probability, the simulator has not chosen this name before for some other file and a query has not been made to the random oracle at name $\| i$ for any $i$.

For each $j, 1 \leq j \leq s$, the simulator chooses random values $\beta_{j}, \gamma_{j} \stackrel{\mathrm{R}}{\leftarrow} \mathbb{Z}_{p}$ and sets $u_{j} \leftarrow g^{\beta_{j}} \cdot h^{\gamma_{j}}$. For each $i, 1 \leq i \leq n$, the simulator chooses a random value $r_{i} \stackrel{R}{\leftarrow} \mathbb{Z}_{p}$, and programs the random oracle at $i$ as

$$
H(\text { name } \| i)=g^{r_{i}} /\left(g^{\sum_{j=1}^{s} \beta_{j} m_{i j}} \cdot h^{\sum_{j=1}^{s} \gamma_{j} m_{i j}}\right) .
$$

Now the simulator can compute $\sigma_{i}$, since we have

$$
\begin{aligned}
H(\text { name } \| i) \cdot \prod_{j=1}^{s} u_{j}^{m_{i j}} & =\prod_{j=1}^{s} u_{j}^{m_{i j}} \cdot g^{r_{i}} / g^{\sum_{j=1}^{s} \beta_{j} m_{i j}} \cdot h^{\sum_{j=1}^{s} \gamma_{j} m_{i j}} \\
& =g^{\sum_{j=1}^{s} \beta_{j} m_{i j}} \cdot h^{\sum_{i=1}^{s} \gamma_{j} m_{i j}} \cdot g^{r_{i}} / g^{\sum_{j=1}^{s} \beta_{j} m_{i j}} \cdot h^{\sum_{j=1}^{s} \gamma_{j} m_{i j}}=g^{r_{i}}
\end{aligned}
$$

so the simulator computes $\sigma_{i}=\left(H(\text { name } \| i) \cdot \prod_{j=1}^{s} u_{j}^{m_{i j}}\right)^{\alpha}=\left(g^{\alpha}\right)^{r_{i}}$.

- The simulator continues interacting with the adversary until the condition specified in the definition of Game 2 occurs: the adversary, as part of a proof-of-retrievability protocol, succeeds in responding with a signature $\sigma^{\prime}$ that is different from the expected signature $\sigma$.

The change made from Game 0 to Game 1 establishes that the parameters associated with this protocol instance - name, $n,\left\{u_{j}\right\},\left\{m_{i j}\right\}$, and $\left\{\sigma_{i}\right\}$ - were generated by the simulator as part of a St query; otherwise, execution would have already aborted. This means that these parameters were generated according to the simulator's procedure described above. Now, dividing the verification equation for the forged signature $\sigma^{\prime}$ by the verification equation for the expected signature $\sigma$, we obtain

$$
e\left(\sigma^{\prime} / \sigma, g\right)=e\left(\prod_{j=1}^{s} u_{j}^{\Delta \mu_{j}}, v\right)=e\left(\prod_{j=1}^{s}\left(g^{\beta_{j}} \cdot h^{\gamma_{j}}\right)^{\Delta \mu_{j}}, v\right)
$$

Rearranging terms yields

$$
e\left(\sigma^{\prime} \cdot \sigma^{-1} \cdot v^{-\sum_{j=1}^{s} \beta_{j} \Delta \mu_{j}}, g\right)=e(h, v)^{\sum_{j=1}^{s} \gamma_{j} \Delta \mu_{j}},
$$

Noting that $v$ equals $g^{\alpha}$, we see that we have found the solution to the computational DiffieHellman problem,

$$
h^{\alpha}=\left(\sigma^{\prime} \cdot \sigma^{-1} \cdot v^{-\sum_{j=1}^{s} \beta_{j} \Delta \mu_{j}}\right)^{\sum_{j=1}^{s} \gamma_{j} \Delta \mu_{j}},
$$


unless evaluating the exponent causes a divide-by-zero. However, we noted already that not all of $\left\{\Delta \mu_{j}\right\}$ can be zero, and the values of $\left\{\gamma_{j}\right\}$ are information theoretically hidden from the adversary ${ }^{20}$ so the denominator is zero only with probability $1 / p$, which is negligible.

Thus if there is a nonnegligible difference between the adversary's probabilities of success in Games 1 and 2, we can construct a simulator that uses the adversary to solve computational Diffie-Hellman, as required.

Game 3. Game 3 is the same as Game 2, with one difference. As before, the challenger tracks St queries and observes proof-of-retrievability protocol instances. This time, if in any of these instances the adversary is successful (i.e., $\mathcal{V}$ outputs 1 ) but at least one of the aggregate messages $m_{j}$ is not equal to the expected $\sum_{\left(i, \nu_{i}\right) \in Q} \nu_{i} m_{i j}$ (where, again, $Q$ is the challenge issued by the verifier) the challenger declares failure and aborts.

Again, let us establish some notation. Suppose the file that causes the abort is $n$ blocks long, has name name, has generating exponents $\left\{u_{j}\right\}$, and contains sectors $\left\{m_{i j}\right\}$, and that the block signatures issued by St are $\left\{\sigma_{i}\right\}$. Suppose $Q=\left\{\left(i, \nu_{i}\right)\right\}$ is the query that causes the challenger to abort, and that the adversary's response to that query was $\mu_{1}^{\prime}, \ldots, \mu_{s}^{\prime}$ together with $\sigma^{\prime}$. Let the expected response - i.e., the one that would have been obtained from an honest prover - be $\mu_{1}, \ldots, \mu_{s}$ and $\sigma$, where $\sigma=\prod_{\left(i, \nu_{i}\right) \in Q} \sigma_{i}^{\nu_{i}}$ and $\mu_{j}=\sum_{\left(i, \nu_{i}\right) \in Q} \nu_{i} m_{i j}$ for $1 \leq j \leq s$. Game 2 already guarantees that we have $\sigma^{\prime}=\sigma$; it is only the values $\left\{\mu_{j}^{\prime}\right\}$ and $\left\{\mu_{j}\right\}$ that can differ. Define $\Delta \mu_{j} \stackrel{\text { def }}{=} \mu_{j}^{\prime}-\mu_{j}$ for $1 \leq j \leq s$; again, at least one of $\left\{\Delta \mu_{j}\right\}$ is nonzero.

We now show that if there is a nonnegligible difference in the adversary's success probabilities between Games 2 and 3 we can construct a simulator that solves the discrete logarithm problem.

The simulator is given as inputs values $g, h \in G$; its goal is to output $x$ such that $h=g^{x}$. The simulator behaves like the Game 2 challenger, with the following differences:

- When asked to store some file whose coded representation comprises the $n$ blocks $\left\{m_{i j}\right\}$, $1 \leq i \leq n, 1 \leq j \leq s$, the simulator behaves according to St, except that For each $j$, $1 \leq j \leq s$, the simulator chooses random values $\beta_{j}, \gamma_{j} \stackrel{\mathrm{R}}{\leftarrow} \mathbb{Z}_{p}$ and sets $u_{j} \leftarrow g^{\beta_{j}} \cdot h^{\gamma_{j}}$.

- The simulator continues interacting with the adversary until the condition specified in the definition of Game 3 occurs: the adversary, as part of a proof-of-retrievability protocol, succeeds in responding with aggregate messages $\left\{\mu_{j}^{\prime}\right\}$ that are different from the expected aggregate messages $\left\{\mu_{j}\right\}$.

As before, we know because of the change made in Game 1 that the parameters associated with this protocol instance were generated by the simulator as part of a St query. Because of the change made in Game 2 we know that $\sigma^{\prime}=\sigma$. Equating the verification equations using $\left\{\mu_{j}^{\prime}\right\}$ and $\left\{\mu_{j}\right\}$ gives us

$$
e\left(\prod_{\left(i, \nu_{i}\right) \in Q} H(\text { name } \| i)^{\nu_{i}} \cdot \prod_{j=1}^{s} u_{j}^{\mu_{j}}, v\right)=e(\sigma, g)=e\left(\sigma^{\prime}, g\right)=e\left(\prod_{\left(i, \nu_{i}\right) \in Q} H(\text { name } \| i)^{\nu_{i}} \cdot \prod_{j=1}^{s} u_{j}^{\mu_{j}^{\prime}}, v\right)
$$

\footnotetext{
${ }^{20}$ Hidden because they are used to compute only the values $\left\{u_{j}\right\}$ in the adversary's view, and these are Pedersen commitments and so information-theoretically hiding.
} 
from which we conclude that

$$
\prod_{j=1}^{s} u_{j}^{\mu_{j}}=\prod_{j=1}^{s} u_{j}^{\mu_{j}^{\prime}}
$$

and therefore that

$$
1=\prod_{j=1}^{s} u_{j}^{\Delta \mu_{j}}=\prod_{j=1}^{s}\left(g^{\beta_{j}} \cdot h^{\gamma_{j}}\right)^{\Delta \mu_{j}}=g^{\sum_{j=1}^{s} \beta_{j} \Delta \mu_{j}} \cdot h^{\sum_{j=1}^{s} \gamma_{j} \Delta \mu_{j}}
$$

We see that we have found the solution to the discrete logarithm problem,

$$
h=g^{-\frac{\sum_{j=1}^{s} \beta_{j} \Delta \mu_{j}}{\sum_{j=1}^{s} \gamma_{j} \Delta \mu_{j}}},
$$

unless the denominator is zero. However, not all of $\left\{\Delta \mu_{j}\right\}$ can be zero, and the values of $\left\{\gamma_{j}\right\}$ are information theoretically hidden from the adversary, so the denominator is zero only with probability $1 / p$, which is negligible.

Thus if there is a nonnegligible difference between the adversary's probabilities of success in Games 2 and 3 , we can construct a simulator that uses the adversary to compute discrete logarithms, as required.

Wrapping up. In Game 3, the adversary is constrained from answering any verification query with values other than those that would have been computed by Pub.P. Yet we have argued that, assuming the signature scheme is secure and computational Diffie-Hellman and discrete logarithm are hard in bilinear groups, there is only a negligible difference in the success probability of the adversary in this game compared to Game 1, where the adversary is not constrained in this manner. Moreover, the hardness of the $\mathrm{CDH}$ problem implies the hardness of the discrete logarithm problem. This completes the proof of Theorem 4.2 .

\subsection{Part-Two Proof}

We say that a cheating prover $\mathcal{P}^{\prime}$ is well-behaved if it never causes $\mathcal{V}$ to accept in a proof-ofretrievability protocol instance except by responding with values $\left\{\mu_{j}\right\}$ and $\sigma$ that are computed correctly, i.e., as they would be by Pub.P. The part-one proofs above guarantee that all adversaries that win the soundness game with nonnegligible probability output cheating provers that are wellbehaved, provided that the cryptographic primitives we employ are secure. The part-two theorem shows that extraction always succeeds against a well-behaved cheating prover:

Theorem 4.3. Suppose a cheating prover $\mathcal{P}^{\prime}$ on an $n$-block file $M$ is well-behaved in the sense above, and that it is $\epsilon$-admissible: i.e., convincingly answers and $\epsilon$ fraction of verification queries. Let $\omega=1 / \# B+(\rho n)^{l} /(n-l+1)^{l}$. Then, provided that $\epsilon-\omega$ is positive and nonnegligible, it is possible to recover a $\rho$ fraction of the encoded file blocks in $O(n /(\epsilon-\omega))$ interactions with $\mathcal{P}^{\prime}$ and in $O\left(n^{2} s+\left(1+\epsilon n^{2}\right)(n) /(\epsilon-\omega)\right)$ time overall.

We first make the following definition. 
Definition 4.4. Consider an adversary $\mathcal{B}$, implemented as a probabilistic polynomial-time Turing machine, that, given a query $Q$ on its input tape, outputs either the correct response (q $M$ in vector notation) or a special symbol $\perp$ to its output tape. Suppose $\mathcal{B}$ responds with probability $\epsilon$, i.e., on an $\epsilon$ fraction of the query-and-randomness-tape space. We say that such an adversary is $\epsilon$-polite.

The proof of our theorem depends upon the following lemma that is proved below.

Lemma 4.5. Suppose that $\mathcal{B}$ is an $\epsilon$-polite adversary as defined above. Let $\omega$ equal $1 / \# B+$ $(\rho n)^{l} /(n-l+1)^{l}$. If $\epsilon>\omega$ then it is possible to recover a $\rho$ fraction of the encoded file blocks in $O(n /(\epsilon-\omega))$ interactions with $\mathcal{B}$ and in $O\left(n^{2} s+\left(1+\epsilon n^{2}\right)(n) /(\epsilon-\omega)\right)$ time overall.

To apply Lemma 4.5, we need only show that a well-behaved $\epsilon$-admissible cheating prover $\mathcal{P}^{\prime}$, as output by a setup-game adversary $\mathcal{A}$, can be turned into an $\epsilon$-polite adversary $\mathcal{B}$. But this is quite simple. Here is how $\mathcal{B}$ is implemented. We will use the $\mathcal{P}^{\prime}$ to construct the $\epsilon$-adversary $\mathcal{B}$. Given a query $Q$, interact with $\mathcal{P}^{\prime}$ according to $\left(\mathcal{V}(p k, s k, t, s k) \rightleftharpoons \mathcal{P}^{\prime}\right)$, playing the part of the verifier. If the output of the interaction is 1 , write $\left(\mu_{1}, \ldots, \mu_{s}\right)$ to the output tape; otherwise, write $\perp$. Each time $\mathcal{B}$ runs $\mathcal{P}^{\prime}$, it provides it with a clean scratch tape and a new randomness tape, effectively rewinding it. Since $\mathcal{P}^{\prime}$ is well-behaved, a successful response will compute $\left(\mu_{1}, \ldots, \mu_{s}\right)$ as prescribed for an honest prover. Since $\mathcal{P}^{\prime}$ is $\epsilon$-admissible, on an $\epsilon$ fraction of interactions it answers correctly. Thus algorithm $\mathcal{B}$ that we have constructed is an $\epsilon$-polite advesrary.

All that remains to to guarantee that $\omega=1 / \# B+(\rho n)^{l} /(n-l+1)^{l}$ is such that $\epsilon-\omega$ is positive - indeed, nonnegligible. But this simply requires that each of $1 / \# B$ and $(\rho n)^{l} /(n-l+1)^{l}$ be negligible in the security parameter; see Section 1.1 .

To prove Lemma 4.5, we must first introduce some arguments in linear algebra.

For a subspace $\mathbb{D}$ of $\left(\mathbb{Z}_{p}\right)^{n}$, denote the dimension of $\mathbb{D}$ by $\operatorname{dim} \mathbb{D}$. Furthermore, let the free variables of a space, free $\mathbb{D}$, be the indices of the basis vectors $\left\{\mathbf{u}_{i}\right\}$ included in $\mathbb{D}$, i.e.,

$$
\text { free } \mathbb{D} \stackrel{\text { def }}{=}\left\{i \in[1, n]: \mathbb{D} \cap \mathbf{u}_{i}=\mathbf{u}_{i}\right\} .
$$

Observe that if we represent $\mathbb{D}$ by means of a basis matrix in row-reduced echelon form, then we can efficiently compute $\operatorname{dim} \mathbb{D}$ and free $\mathbb{D}$.

Next, we give two claims.

Claim 4.6. Let $\mathbb{D}$ be a subspace of $\left(\mathbb{Z}_{p}\right)^{n}$, and let $I$ be an l-element subset of $[1, n]$. If $I \nsubseteq$ free $\mathbb{D}$, then a random query over indices $I$ with coefficients in $B$ is in $\mathbb{D}$ with probability at most $1 / \# B$.

Proof. Let $\mathbb{I}$ be the subspace spanned by the unit vectors in $I$, i.e., by $\left\{\mathbf{u}_{i}\right\}_{i \in I}$. Clearly, $\operatorname{dim} \mathbb{D} \cap \mathbb{I}$ is at most $l-1$; if it equalled $l$, then we would have $\mathbb{D} \cap \mathbb{I}=\mathbb{I}$ and each of the vectors $\left\{\mathbf{u}_{i}\right\}_{i \in I}$ would be in $\mathbb{D}$, contradicting the lemma statement. Suppose $\operatorname{dim} \mathbb{D} \cap \mathbb{I}$ equals $r$. Then there exist $r$ indices in $I$ such that a choice of values for the coordinates at these indices determines the values of the remaining $l-r$ coordinates. This means that there are at most $(\# B)^{r}$ vectors in $\mathbb{D} \cap \mathbb{I}$ with coordinate values in $B$ : a choice of one of $\# B$ values for each of the $r$ coordinates above determines the value to each of the other $l-r$ coordinates; if the values of these coordinates are all in $B$, then this vector contributes 1 to the count; otherwise it contributes 0 . The maximum possible count is thus $(\# B)^{r}$. By contrast, there are $(\# B)^{l}$ vectors in $\mathbb{I}$ with coordinates in $B$, and these are exactly the vectors corresponding to each random query with indices $I$. Thus the probability that a random query is in $\mathbb{D}$ is at most $1 /(\# B)^{l-r} \leq 1 /(\# B)$, which proves the lemma. 
Claim 4.7. Let $\mathbb{D}$ be a subspace of $\left(\mathbb{Z}_{p}\right)^{n}$, and suppose that $\#($ free $\mathbb{D})=m$. Then for a random l-element subset $I$ of $[1, n]$ the probability that $I \subseteq$ free $\mathbb{D}$ is at most $m^{l} /(n-l+1)^{l}$.

Proof. Color the $m$ indices included in free $\mathbb{D}$ black; color the remaining $n-m$ indices white. A query $I$ corresponds to a choice of $l$ indices out of all these, without replacement. A query satisfies the condition that $I \subseteq$ free $\mathbb{D}$ exactly if every element of $I$ is in free $\mathbb{D}$, i.e., is colored black. Thus the probability that a random query satisfies the condition is just the probability of drawing $l$ black balls, without replacement, from a jar containing $m$ black balls and $n-m$ white balls; and this probability is

$$
\left(\begin{array}{c}
m \\
l
\end{array}\right) /\left(\begin{array}{l}
n \\
l
\end{array}\right)=\frac{(m ! /(m-l) !)}{(n ! /(n-l) !)}<\frac{m^{l}}{(n-l+1)^{l}},
$$

as required. Note that if $m>n-l$ then we will have $I \subseteq$ free $\mathbb{D}$ with probability 1 ; the upper bound given by this lemma is a probability greater than 1 in this case, but of course still correct.

Lemma 4.5. Suppose that $\mathcal{B}$ is an $\epsilon$-polite adversary as defined above. Let $\omega$ equal $1 / \# B+$ $(\rho n)^{l} /(n-l+1)^{l}$. If $\epsilon>\omega$ then it is possible to recover a $\rho$ fraction of the encoded file blocks in $O(n /(\epsilon-\omega))$ interactions with $\mathcal{B}$ and in $O\left(n^{2} s+\left(1+\epsilon n^{2}\right)(n) /(\epsilon-\omega)\right)$ time overall.

Proof. We say the extractor's knowledge at each point is a subspace $\mathbb{D}$, represented by a $t \times n$ matrix $A$ in row-reduced echelon form. Suppose that the query-response pairs contributing to the extractor's knowledge are

$$
\mathbf{q}^{(1)} M=\left(\mu_{1}^{(1)}, \ldots, \mu_{s}^{(1)}\right) \quad \ldots \quad \mathbf{q}^{(t)} M=\left(\mu_{1}^{(t)}, \ldots, \mu_{s}^{(t)}\right),
$$

or $V M=W$, where $V$ is the $t \times n$ matrix whose rows are $\left\{\mathbf{q}^{(i)}\right\}$ and $W$ is the $t \times s$ matrix whose rows are $\left(\mu_{1}^{(i)}, \ldots, \mu_{s}^{(i)}\right)$. The row-reduced echelon matrix $A$ is related to $V$ by $A=U V$, where $U$ is a $t \times t$ matrix with nonzero determinant computed in applying Gaussian elimination to $V$.

The extractor's knowledge is initially empty, i.e., $\mathbb{D}=\emptyset$.

The extractor repeats the following behavior until \#(free $\mathbb{D}) \geq \rho n$ :

The extractor chooses a random query $Q$. It runs $\mathcal{B}$ on $Q$. Suppose $\mathcal{B}$ chooses to respond, giving answer $\left(\mu_{1}, \ldots, \mu_{s}\right)$; clearly this happens with probability $\epsilon$. Let $Q$ be over indices $I \in[1, n]$, and denote it in vector notation as $\mathbf{q}$. Now we classify $Q$ into three types:

1. $\mathbf{q} \notin \mathbb{D}$;

2. $\mathbf{q} \in \mathbb{D}$ but $I \nsubseteq$ free $\mathbb{D}$; or

3. $I \subseteq$ free $\mathbb{D}$.

For queries of the first type, the extractor adds $Q$ to its knowledge $\mathbb{D}$, obtaining new knowledge $\mathbb{D}^{\prime}$, as follows. It adds a row corresponding to the query to $V$, obtaining $V^{\prime}$, and a row corresponding to the response to $W$, obtaining $W^{\prime}$; it modifies the transform matrix $U$, obtaining $U^{\prime}$, so that $A^{\prime}=U^{\prime} V^{\prime}$ is again in row-reduced echelon form and spans q. The primed versions $\mathbb{D}^{\prime}, A^{\prime}, U^{\prime}, V^{\prime}$, and $W^{\prime}$ replace the unprimed versions in the extractor's state. For queries of type 2 or 3 , the extractor does not add to its knowledge. Regardless, the extractor continues with another query.

Clearly, a type- 1 query increases $\operatorname{dim} \mathbb{D}$ by 1 . If $\operatorname{dim} \mathbb{D}$ equals $n$ then free $\mathbb{D}=[1, n]$ and $\#($ free $\mathbb{D})=n \geq \rho n$, so the extractor's query phase is guaranteed to terminate by the time it has encountered $n$ type- 1 queries. 
We now observe that any time the simulator is in its query phase, type- 1 queries make up at least a $1-\omega$ fraction of the query space. By Claim 4.6, type-2 queries make up at most a $(1 / \# B)$ fraction of the query space, since

$$
\begin{aligned}
\operatorname{Pr}_{Q}[Q \text { is type- } 2] & =\operatorname{Pr}_{Q}[\mathbf{q} \in \mathbb{D} \wedge I \nsubseteq \text { free } \mathbb{D}] \\
& =\operatorname{Pr}_{Q}[\mathbf{q} \in \mathbb{D} \mid I \nsubseteq \text { free } \mathbb{D}] \cdot \operatorname{Pr}_{Q}[I \nsubseteq \text { free } \mathbb{D}] \\
& \leq \operatorname{Pr}_{Q}[\mathbf{q} \in \mathbb{D} \mid I \nsubseteq \text { free } \mathbb{D}] \\
& \leq 1 / \# B,
\end{aligned}
$$

where it is the last inequality that follows from the claim ${ }^{21}$ Here the probability expressions are all over a random choice of query $Q$, and $I$ and $\mathbf{q}$ are the index set and vector form corresponding to the chosen query.

Similarly, suppose that $\#($ free $\mathbb{D})=m$. Then by Claim 4.7, type-3 queries make up at most an $m^{l} /(n-l+1)^{l}$ fraction of the query space, and since $m<\rho n$ (otherwise the extractor would have ended the query phas) this fraction is at most $(\rho n)^{l} /(n-l+1)^{l}$.

Therefore the fraction of the query space consisting of type- 2 and type- 3 queries is at most $1 / \# B+(\rho n)^{l} /(n-l+1)^{l}=\omega$. Since query type depends on the query and not on the randomness supplied to $\mathcal{B}$, it follows that the fraction of query-and-randomness-tape space consisting of type-1 and type- 2 queries is also at most $\omega$. Now, $\mathcal{B}$ must respond correctly on an $\epsilon$ fraction of the queryand-randomness-tape space. Even if the adversary is as unhelpful as it can be and this $\epsilon$ fraction includes the entire $\omega$ fraction of type- 2 and type- 3 queries, there remains at least an $(\epsilon-\omega)$ fraction of the query-and-randomness-tape space to which the adversary will respond correctly and in which the query is of type 1 and therefore helpful to the extractor. (By assumption $\epsilon>\omega$, so this fraction is nonempty.)

Since the extractor needs at most $n$ successful type- 1 queries to complete the query phase and it obtains a successful type- 1 query from an interaction with $\mathcal{B}$ with probability $O(\epsilon-\omega)$, it follows that the extractor will require at most $O(n /(\epsilon-\omega))$ interactions.

With $\mathbb{D}$ represented by a basis matrix $A$ in row-reduced echelon form, it is possible, given a query $\mathbf{q}$ to which the adversary has responded, to determine efficienty which type it is. The extractor appends q to $A$ and runs the Gaussian elimination algorithm on the new row, a process that takes $O\left(n^{2}\right)$ time [10, Section 2.3] ${ }^{22}$ If the reduced row is not all zeros then the query is type 1 ; the reduction also means that the augmented matrix $A^{\prime}$ is again in row-reduced echelon form, and the steps of the reduction also give the appropriate updates to the transform matrix $U^{\prime}$. Since the reduction need only be performed for the $\epsilon$ fraction of queries to which $\mathcal{B}$ correctly responds, the overall running time of the query phase is $O\left(\left(1+\epsilon n^{2}\right)(n) /(\epsilon-\omega)\right)$.

Once the query phase is complete, the extractor has matrices $A, U, V$, and $W$ such that $V M=W$ (where $M=\left(m_{i j}\right)$ is the matrix consisting of encoded file blocks), $A=U V$, and $A$ is in row-reduced echelon form. Moreover, there are at least $\rho n$ free dimensions in the subspace $\mathbb{D}$ spanned by $A$ and by $V$. Suppose $i$ is in free $\mathbb{D}$. Since $A$ is in row-reduced echelon form, there must be a row in $A$, say row $t$, that equals the $i$ th basis vector $\mathbf{u}_{i}$. Multiplying both sides of

\footnotetext{
${ }^{21}$ The claim gives a condition for a single $I$ satisfying the condition $I \nsubseteq$ free $\mathbb{D}$; the inequality here is over all such $I$; but if the probability never exceeds $1 / \# B$ for any specific $I$ then it doesn't exceed $1 / \# B$ over a random choice of $I$, either.

${ }^{22}$ More specifically, $O(t n)$ time if $A$ is a $t \times n$ matrix; but of course $t \leq n$.
} 
$V M=W$ by $U$ on the left gives the equation $A M=U W$. For any $j, 1 \leq j \leq s$, consider the entry at row $t$ and column $j$ in the matrix $A M$. It is equal to $\mathbf{u}_{i} \cdot\left(m_{1, j}, m_{2, j}, \ldots, m_{n, j}\right)=m_{i, j}$. If we compute the matrix product $U W$, we can thus read off from it every block of every sector for $i \in$ free $\mathbb{D}$. Computing the matrix multiplication takes $O\left(n^{2} s\right)$ time. The extractor computes the relevant rows, outputs them, and halts.

Note that while we have described the extraction algorithm as performing row reduction operations, it could instead collect $n$ successful interactions with the cheating prover and then perform a single Gaussian elimination using an algorithm specialized for sparse matrices, reducing the asymptotic runtime substantially. We do not expect that the extraction algorithm will be used in actual outsourced storage deployments, so this improvement is not important in practice. This completes the proof of Lemma 4.5 .

\subsection{Part-Three Proof}

Theorem 4.8. Given a $\rho$ fraction of the $n$ blocks of an encoded file $M^{*}$, it is possible to recover the entire original file $M$ with all but negligible probability.

Proof. For rate- $\rho$ Reed-Solomon codes this is trivially true, since any $\rho$ fraction of encoded file blocks suffices for decoding; see Appendix A. For rate- $\rho$ linear-time codes the additional measures described in Appendix A.1 guarantee that the $\rho$ fraction of blocks retrieved will allow decoding with overwhelming probability.

\section{Proof for the Simple MAC Scheme}

In this section we recall the simple MAC scheme described by Naor and Rothblum [25] and Juels and Kaliski [20] and give a formal proof for its security in the proof-of-retrievability model. We use the same common notation as in Section 3.1.

\subsection{The Construction}

Let $f:\{0,1\}^{*} \times \mathcal{K}_{\text {prf }} \rightarrow \mathbb{Z}_{p}$ be a PRF. The construction of the simple scheme Simple is:

Simple. $K \mathbf{g}()$. Choose a random MAC key $k_{\text {mac }} \stackrel{R}{\leftarrow} \mathcal{K}_{\text {mac }}$. The secret key is $s k=\left(k_{\text {mac }}\right)$; there is no public key.

Simple.St $(s k, M)$. Given the file $M$, first apply the erasure code to obtain $M^{\prime}$; then split $M^{\prime}$ into $n$ blocks (for some $n$ ), each $s$ sectors long: $\left\{m_{i j}\right\}_{\substack{1 \leq i \leq n \\ 1 \leq j \leq s}}$. Choose a random file name name from some sufficiently large domain (e.g., $\mathbb{Z}_{p}$ ). The file tag is $t=($ name). Now, for each $i, 1 \leq i \leq n$, compute

$$
\sigma_{i} \leftarrow \operatorname{MAC}_{k_{\text {mac }}}\left(\text { name }\|i\| m_{i 1}\|\cdots\| m_{i s}\right) .
$$

The processed file $M^{*}$ is $\left\{m_{i j}\right\}, 1 \leq i \leq n, 1 \leq j \leq s$ together with $\left\{\sigma_{i}\right\}, 1 \leq i \leq n$.

Simple. $\mathcal{V}(p k, s k, t)$. Parse $s k$ as $\left(k_{\mathrm{mac}}\right)$. Parse $t$ as (name). Pick a random $l$-element subset $I$ of the set $[1, n]$. Send $I$ to the prover. 
Parse the prover's response to obtain $m_{i 1}, \ldots, m_{i s}$ and $\sigma_{i}$, all in $\mathbb{Z}_{p}$, for each $i \in I$. If parsing fails, fail by emitting 0 and halting. Otherwise, check for each $i \in I$ whether

$$
\sigma_{i} \stackrel{?}{=} \mathrm{MAC}_{k_{\mathrm{mac}}}\left(\text { name }\|i\| m_{i 1}\|\cdots\| m_{i s}\right) ;
$$

if all $l$ equations hold, output 1 ; otherwise, output 0 .

Simple. $\mathcal{P}\left(p k, t, M^{*}\right)$. Parse the processed file $M^{*}$ as $\left\{m_{i j}\right\}, 1 \leq i \leq n, 1 \leq j \leq s$, along with $\left\{\sigma_{i}\right\}$, $1 \leq i \leq n$. Parse the message sent by the verifier as $I$, an $l$-element subset of $[1, n]$. Send to the prover, for each $i \in I$, the values $m_{i 1}, \ldots, m_{i s}$ and $\sigma_{i}$.

Note that it is easy to modify the scheme and the proof to use a signature scheme instead of a MAC to obtain public verifiability.

\subsection{The Proof}

Theorem 5.1. If the MAC scheme is unforgeable then (except with negligible probability) no adversary against the soundness of the simple scheme ever causes $\mathcal{V}$ to accept in a proof-of-retrievability protocol instance, except by responding with values $\left\{m_{i j}\right\}$ and $\left\{\sigma_{i}\right\}$ that are computed correctly, i.e., as they would be by Simple.P.

Proof. The simulator is given oracle access to the MAC; its goal is to create a forgery. The simulator plays the part of the environment in interacting with the attacker, using its MACgeneration oracle to create the $\left\{\sigma_{i}\right\}$ MACs. Whenever the adversary responds in a proof-of-storage protocol instance where name is not one of the names issued by the simulator in a store query, the simulator uses its MAC verification oracle to check whether any $\sigma_{i}$ sent by the adversary, for $i \in I$, is a valid MAC ${ }^{23}$ Such a valid MAC would be a forgery, since the simulator never requests a MAC on a name not chosen in a store query. Whenever the adversary responds in a proof-ofstorage protocol instance on a file with tag name whose blocks are $\left\{m_{i j}\right\}$ and where, for some $i \in$ $I$, the values $\left\{m_{i j}^{\prime}\right\}_{j}$ sent by the adversary are different from the values $\left\{m_{i j}\right\}_{j}$ in the file, the simulator uses its MAC verification oracle to check whether the corresponding authenticator is valid. Such a valid MAC would be a forgery, since the simulator never requested a MAC on any string beginning "name $\|i\| \cdots$ " except for "name $\|i\| m_{i 1}\|\cdots\| m_{i s}$ ", and $\left(m_{i 1}, \ldots, m_{i s}\right) \neq\left(m_{i 1}^{\prime}, \ldots, m_{i s}^{\prime}\right)$ by assumption. (Because name is drawn from a large space, each file storage query will use a different value for name, except with negligible probability.) We see that if the adversary ever causes $\mathcal{V}$ to accept in a proof-of-retrievability protocol instance without responding with values $\left\{m_{i j}\right\}$ and $\left\{\sigma_{i}\right\}$ computed as they would be by Simple.P , the simulator finds a MAC forgery.

As before, we say that a cheating prover $\mathcal{P}^{\prime}$ is well-behaved if it never causes $\mathcal{V}$ to accept in a proof-of-retrievability protocol instance except by responding with values $\left\{m_{i j}\right\}$ and $\left\{\sigma_{i}\right\}$ that are computed correctly, i.e., as they would be by Simple.P. The theorem above guarantee that all adversaries that win the soundness game with nonnegligible probability output cheating provers that are well-behaved, provided that the MAC we employ is secure. The next theorem shows that extraction always succeeds against a well-behaved cheating prover:

\footnotetext{
${ }^{23}$ See Bellare, Goldreich, and Mityagin [7] for why the MAC security definition incorporates verification queries.
} 
Theorem 5.2. Suppose a cheating prover $\mathcal{P}^{\prime}$ on an $n$-block file $M$ is well-behaved in the sense above, and that it is $\epsilon$-admissible: i.e., convincingly answers and $\epsilon$ fraction of verification queries. Then, provided that $\epsilon-(\rho n)^{l} /(n-l+1)^{l}$ is positive and nonnegligible, it is possible to recover a $\rho$ fraction of the encoded file blocks in $O\left(\rho n /\left(\epsilon-(\rho n)^{l} /(n-l+1)^{l}\right)\right)$ interactions with $\mathcal{P}^{\prime}$.

Proof. We turn the $\epsilon$-admissible, well-behaved cheating prover $\mathcal{P}^{\prime}$ into an $\epsilon$-polite adversary $\mathcal{B}$ as in the proof of Theorem 4.3, by interacting with $\mathcal{P}^{\prime}$, checking the MACs $\left\{\sigma_{i}\right\}$ on each block $i \in I$, and emitting $\left\{m_{i j}\right\}$ if all $l$ MACS are valid, $\perp$ otherwise.

Against an $\epsilon$-polite adversary the extractor works as follows. Its knowledge is a subset $S \subseteq[1, n]$, initially empty. If $\# S$ ever reaches $\rho n$, the extractor halts. The extractor repeatedly chooses a random $l$-element query $I \subset[1, n]$ and sends $I$ to the polite adversary $\mathcal{B}$. If the adversary does not output $\perp$, the extractor updates its knowledge as $S^{\prime} \leftarrow S \cup I$. Regardless, the extractor continues with another query.

A query is answers as not $\perp$ with probability $\epsilon$. For such a query, $\# S$ increases by at least 1 provided that $I \nsubseteq S$. But if the extractor has not yet halted we have $\# S<\rho n$, and the probability that a random $l$-element subset $I$ of $[1, n]$ is such that $I \subseteq S$ is at most $(\rho n)^{l} /(n-l+1)^{l}$, by reasoning identical to that used in the proof of Lemma 4.7. This means that on an $\epsilon-(\rho n)^{l} /(n-l+1)^{l}$ fraction of the query-randomness space the adversary $\mathcal{B}$ will give a response that increases \#S. Thus $O\left(\rho n /\left(\epsilon-(\rho n)^{l} /(n-l+1)^{l}\right)\right)$ interactions with the adversary suffice to grow \#S to $\rho n$ elements, at which point the extractor halts.

But each element $i \in S$ corresponds to a block $i$ for which the extractor has learned the values $m_{i 1}, \ldots, m_{i s}$ (since the adversary is polite), so the extractor will have recovered $\rho n$ blocks of the file, as required.

Theorem 5.3. Given a $\rho$ fraction of the $n$ blocks of an encoded file $M^{*}$, it is possible to recover the entire original file $M$ with all but negligible probability.

Proof. For rate- $\rho$ Reed-Solomon codes this is trivially true, since any $\rho$ fraction of encoded file blocks suffices for decoding; see Appendix A. For rate- $\rho$ linear-time codes the additional measures described in Appendix A.1 guarantee that the $\rho$ fraction of blocks retrieved will allow decoding with overwhelming probability.

\section{Construction with RSA Signatures}

In this section, we show how the RSA construction of Ateniese et al. 3] can be considered an instantiation of our framework for proofs of retrievability. The construction closely follows that of Ateniese et al., and the part-one proof also uses RSA techniques similar to theirs [4, Theorem 4.3]. The benefit of this section is to show that an RSA-based construction very similar to that of Ateniese et al. admits a full and rigorous proof of security.

\subsection{Construction}

Let $\lambda$ be the security parameter, and let $\lambda_{1}$ be a bitlength such that the difficulty of factoring a $\left(2 \lambda_{1}-1\right)$-bit modulus is appropriate to the security parameter $\lambda$. Let $\max B$ be the largest element in $B$, and let $\lambda_{2}$ be a bitlength equal to $\left.\lg (l \cdot \max B)\right\rceil+1$.

The construction of the private verification scheme PubRSA is: 
PubRSA.Kg(). Generate a random signing keypair (spk, ssk) $\stackrel{\mathrm{R}}{\leftarrow} \mathrm{SKg}$. Choose two random primes $p$ and $q$ in the range $\left[2^{\lambda_{1}-1}, 2^{\lambda_{1}}-1\right]$. Let $N=p q$ be the RSA modulus; we have $2^{2 \lambda_{1}-2}<$ $N<2^{2 \lambda_{1}}$. Let $H:\{0,1\}^{*} \rightarrow \mathbb{Z}_{N}^{*}$ be a full-domain hash, which we treat as a random oracle ${ }^{24}$ Choose a random $2 \lambda_{1}+\lambda_{2}$-bit prime $e$, and set $d=e^{-1} \bmod \phi(N)$. The secret key is $s k=(N, d, H, s s k)$; the public key is $p k=(N, e, H, s p k)$.

PubRSA.St $(s k, M)$. Given the file $M$, first apply the erasure code to obtain $M^{\prime}$; then split $M^{\prime}$ into $n$ blocks (for some $n$ ), each $s$ sectors: $\left\{m_{i j}\right\}_{\substack{1 \leq i \leq n \\ 1 \leq j \leq s}}$. Each sector $m_{i j}$ is an element of $\mathbb{Z}_{N}$. Now parse sk as $(N, d, H$, ssk). Choose a random file name name from some sufficiently large domain (e.g., $\left.\mathbb{Z}_{N}\right)$. Choose $s$ random elements $u_{1}, \ldots, u_{s} \stackrel{\mathrm{R}}{\leftarrow} \mathbb{Z}_{N}^{*}$. Let $t_{0}$ be "name $\|n\| u_{1}\|\cdots\| u_{s}$ "; the file tag $t$ is $t_{0}$ together with a signature, on $t_{0}$ under private key ssk: $t \leftarrow t_{0} \| \operatorname{SSig}_{\text {ssk }}\left(t_{0}\right)$.

Now, for each $i, 1 \leq i \leq n$, compute

$$
\sigma_{i} \leftarrow\left(H(\text { name } \| i) \cdot \prod_{j=1}^{s} u_{j}^{m_{i j}}\right)^{d} \bmod N .
$$

The processed file $M^{*}$ is $\left\{m_{i j}\right\}, 1 \leq i \leq n, 1 \leq j \leq s$ together with $\left\{\sigma_{i}\right\}, 1 \leq i \leq n$.

PubRSA.V $\mathcal{V}(p k, s k, t)$. Parse $p k$ as $(N, e, H, s p k)$. Use spk to verify the signature on on $t$; if the signature is invalid, reject by emitting 0 and halting. Otherwise, parse $t$, recovering name, $n$, and $u_{1}, \ldots, u_{s}$. Now pick a random $l$-element subset $I$ of the set $[1, n]$, and, for each $i \in I$, a random element $\nu_{i} \stackrel{\mathrm{R}}{\leftarrow} B$. Let $Q$ be the set $\left\{\left(i, \nu_{i}\right)\right\}$. Send $Q$ to the prover.

Parse the prover's response to obtain $\mu_{1}, \ldots, \mu_{s}$ and $\sigma \in \mathbb{Z}_{N}$. Check that each $\mu_{j}$ is in the range $[0, l \cdot N \cdot \max B]$. If parsing fails or the $\left\{\mu_{j}\right\}$ values are not in range, fail by emitting 0 and halting. Otherwise, check whether

$$
\sigma^{e} \stackrel{?}{=} \prod_{\left(i, \nu_{i}\right) \in Q} H(\text { name } \| i)^{\nu_{i}} \cdot \prod_{j=1}^{s} u_{j}^{\mu_{j}} \bmod N ;
$$

if so, output 1 ; otherwise, output 0 .

PubRSA.P $\left(p k, t, M^{*}\right)$. Parse the processed file $M^{*}$ as $\left\{m_{i j}\right\}, 1 \leq i \leq n, 1 \leq j \leq s$, along with $\left\{\sigma_{1}\right\}, 1 \leq i \leq n$. Parse the message sent by the verifier as $Q$, an $l$-element set $\left\{\left(i, \nu_{i}\right)\right\}$, with the $i$ 's distinct, each $i \in[1, n]$ and each $\nu_{i} \in B$.

For each $j, 1 \leq s \leq j$, compute

$$
\mu_{j} \leftarrow \sum_{\left(i, \nu_{i}\right) \in Q} \nu_{i} m_{i j} \in \mathbb{Z}
$$

where the sum is computed in $\mathbb{Z}$, without modular reduction. In addition, compute

$$
\sigma \leftarrow \prod_{\left(i, \nu_{i}\right) \in Q} \sigma_{i}^{\nu_{i}} \bmod N
$$

Send to the prover in response the values $\mu_{1}, \ldots, \mu_{s}$ and $\sigma$.

\footnotetext{
${ }^{24}$ The hash $H$ can be instantiated using a hash onto all of $\mathbb{Z}_{N}$; a value not in $\mathbb{Z}_{N}^{*}$ would disclose the factorization of $N$ and thus will never appear in practice.
} 


\subsection{Part-One Proof}

We now give the part one proof of our scheme.

We begin with technical observations about $\mathbb{Z}_{N}^{*}$ that will be of use below. For $e$ relatively prime to $\phi(N)$, the map $x \mapsto x^{e} \bmod N$ is an isomorphism of $\mathbb{Z}_{N}^{*}$; since $e$ as chosen above is prime and larger than $N$, it must be relatively prime to $\phi(N)$, as required. For $c \in \mathbb{Z}_{N}^{*}$, the map $x \mapsto c x \bmod N$ is also an isomorphism of $\mathbb{Z}_{N}^{*}$. Thus for $x \in \mathbb{Z}_{N}^{*}$, the value $c x$ for a random $c \in \mathbb{Z}_{N}^{*}$ is information-theoretically independent of $x$. In addition, we will use the following lemma (see [18] and Lemma 1 of [13]):

Lemma 6.1. Given $x, y \in \mathbb{Z}_{N}$, along with $a, b \in \mathbb{Z}$ such that $x^{a}=y^{b}$ and $\operatorname{gcd}(a, b)=1$, one can efficiently compute $\bar{x} \in \mathbb{Z}_{N}$ such that $\bar{x}^{a}=y$.

Theorem 6.2. If the signature scheme used for file tags is existentially unforgeable and the RSA problem with large public exponents is hard, then, in the random oracle model, except with negligible probability no adversary against the soundness of our public-verification scheme ever ever causes $\mathcal{V}$ to accept in a proof-of-retrievability protocol instance, except by responding with values $\left\{\mu_{j}\right\}$ and $\sigma$ that are computed correctly, i.e., as they would be by PubRSA.P.

Once more, we prove the theorem as a series of games.

Game 0. The first game, Game 0, is simply the challenge game defined in Section 2. By assumption, the adversary $\mathcal{A}$ wins with nonnegligible probability.

Game 1. Game 1 is the same as Game 0, with one difference. The challenger keeps a list of all signed tags ever issued as part of a store-protocol query. If the adversary ever submits a tag $t$ either in initiating a proof-of-storage protocol or as the challenge tag, that (1) has a valid signature under ssk but (2) is not a tag signed by the challenger, the challenger declares failure and aborts.

Clearly, if there is a difference in the adversary's success probability between Games 0 and 1, we can use the adversary to construct a forger against the signature scheme.

Game 2. Game 2 is the same as Game 1, with one difference. The challenger keeps a list of its responses to St queries made by the adversary. Now the challenger observes each instance of the proof-of-storage protocol with the adversary - whether because of a proof-of-storage query made by the adversary, or in the test made of $\mathcal{P}^{\prime}$, or as part of the extraction attempt by Extr. If in any of these instances the adversary is successful (i.e., $\mathcal{V}$ outputs 1 ) but either

1. the adversary's aggregate signature $\sigma$ is not equal to $\prod_{\left(i, \nu_{i}\right) \in Q} \sigma_{i}^{\nu_{i}} \bmod N$ (where $Q$ is the challenge issued by the verifier and $\sigma_{i}$ are the signatures on the blocks of the file considered in the protocol instance) or

2. at least one the adversary's aggregate block values $\mu_{1}^{\prime}, \ldots, \mu_{s}^{\prime}$ is not equal to the expected block value $\mu_{j}=\sum_{\left(i, \nu_{i}\right) \in Q} \nu_{i} m_{i j}$,

the challenger declares failure and aborts.

Before analyzing the difference in success probabilities between Games 1 and 2, we will establish some notation and draw a few conclusions. Suppose the file that causes the abort is $n$ blocks long, has name name, has generating exponents $\left\{u_{j}\right\}$, and contains sectors $\left\{m_{i j}\right\}$, and that the block 
signatures issued by St are $\left\{\sigma_{i}\right\}$. Suppose $Q=\left\{\left(i, \nu_{i}\right)\right\}$ is the query that causes the challenger to abort, and that the adversary's response to that query was $\mu_{1}^{\prime}, \ldots, \mu_{s}^{\prime}$ together with $\sigma^{\prime}$. Let the expected response - i.e., the one that would have been obtained from an honest prover - be $\mu_{1}, \ldots, \mu_{s}$ and $\sigma$, where $\sigma=\prod_{\left(i, \nu_{i}\right) \in Q} \sigma_{i}^{\nu_{i}} \bmod N$ and $\mu_{j}=\sum_{\left(i, \nu_{i}\right) \in Q} \nu_{i} m_{i j}$ for $1 \leq j \leq s$. By the correctness of the scheme, we know that the expected response satisfies the verification equation, i.e., that

$$
\sigma^{e}=\prod_{\left(i, \nu_{i}\right) \in Q} H(\text { name } \| i)^{\nu_{i}} \cdot \prod_{j=1}^{s} u_{j}^{\mu_{j}}
$$

Because the challenger aborted, we know that $\sigma^{\prime}$ and $\mu_{1}^{\prime}, \ldots, \mu_{s}^{\prime}$ passed the verification equation, i.e., that

$$
\left(\sigma^{\prime}\right)^{e}=\prod_{\left(i, \nu_{i}\right) \in Q} H(\text { name } \| i)^{\nu_{i}} \cdot \prod_{j=1}^{s} u_{j}^{\mu_{j}^{\prime}} .
$$

(Note that if $\sigma^{\prime}$ is in $\mathbb{Z}_{N} \backslash \mathbb{Z}_{N}^{*}$ then so is $\left(\sigma^{\prime}\right)^{e}$, whereas the right-hand side of the verification equation is in $\mathbb{Z}_{N}^{*}$. Thus the verification equation will not hold unless $\sigma^{\prime}$ is in $\mathbb{Z}_{N}^{*}$, which is why no separate check in $\mathcal{V}$ that is required that $\sigma^{\prime}$ is relatively prime to $N$.)

Now observe that condition 1 , above, implies condition 2, which means that having the simulator abort on either condition 1 or 2 is the same as having it abort on just condition 2: if condition 2 doesn't hold then $\mu_{j}^{\prime}=\mu_{j}$ for each $j$, and it follows from the verification equation that $\left(\sigma^{\prime}\right)^{e}=\sigma^{e}$. Because $\mathcal{V}$ checked that $\sigma^{\prime}$ is in $\mathbb{Z}_{N}$ and because, as noted, the verification equation requires that $\sigma^{\prime}$, like $\sigma$, is in $\mathbb{Z}_{N}^{*}$, the fact that exponentiation by $e$ is an isomorphism of $\mathbb{Z}_{N}^{*}$ means that $\left(\sigma^{\prime}\right)^{e}=\sigma^{e}$ implies $\sigma^{\prime}=\sigma$, so condition 1 doesn't hold, either.

Therefore, if we define $\Delta \mu_{j} \stackrel{\text { def }}{=} \mu_{j}^{\prime}-\mu_{j}$ for $1 \leq j \leq s$, it must be the case that if the simulator aborts at least one of $\left\{\Delta \mu_{j}\right\}$ is nonzero.

With this in mind, we now show that if there is a nonnegligible difference in the adversary's success probabilities between Games 1 and 2 we can construct a simulator that solves the RSA problem when the public exponent $e$ is large.

The simulator is given as inputs a $2 \lambda_{1}$-bit modulus $N$ and a $\left(2 \lambda_{1}+\lambda_{2}\right)$-bit public exponent $e$, along with a value $y \in \mathbb{Z}_{N}^{*}$; its goal is to output $x \in \mathbb{Z}_{N}^{*}$ such that $x^{e}=y$. The simulator behaves like the Game 1 challenger, with the following differences:

- In generating a public key, it sets the modulus and public exponent to $N$ and $e$; it does not know the corresponding secret modulus $d$.

- The simulator programs the random oracle $H$. It keeps a list of queries and responses to answers consistently. In answering the adversary's queries it responds with a random $g \stackrel{\mathrm{R}}{\leftarrow} \mathbb{Z}_{N}^{*}$. The simulator also answers queries of the form $H($ name $\| i)$ in a special way, as we will see below.

- When asked to store some file whose coded representation comprises the $n$ blocks $\left\{m_{i j}\right\}$, $1 \leq i \leq n, 1 \leq j \leq s$, the simulator behaves as follows. It chooses a name name at random. Because the space from which names are drawn is large, it follows that, except with negligible probability, the simulator has not chosen this name before for some other file and a query has not been made to the random oracle at name $\| i$ for any $i$. 
For each $j, 1 \leq j \leq s$, the simulator chooses a random $g_{j} \stackrel{\mathrm{R}}{\leftarrow} \mathbb{Z}_{N}^{*}$ and $\beta_{j} \stackrel{\mathrm{R}}{\leftarrow}\left[1,2^{\lambda}\right]$ and sets $u_{j} \leftarrow g_{j}^{e} y^{\beta_{j}}$. For each $i, 1 \leq i \leq n$, the simulator chooses a random value $h_{i} \stackrel{\mathrm{R}}{\leftarrow} \mathbb{Z}_{N}^{*}$, and programs the random oracle at $i$ as

$$
H(\text { name } \| i)=h_{i}^{e} / \prod_{j=1}^{s} u_{j}^{m_{i j}} .
$$

Now the simulator can compute $\sigma_{i}$, since we have

$$
H(\text { name } \| i) \cdot \prod_{j=1}^{s} u_{j}^{m_{i j}}=h_{i}^{e}
$$

if the simulator sets $\sigma_{i}=h_{i}$, we will have $\sigma_{i}^{e}=h_{i}^{e \cdot r_{i}}=H($ name $\| i) \cdot \prod_{j=1}^{s} u_{j}^{m_{i j}}$, as required.

- The simulator continues interacting with the adversary until the condition specified in the definition of Game 2 occurs: the adversary, as part of a proof-of-storage protocol, succeeds in responding with a signature $\sigma^{\prime}$ that is different from the expected signature $\sigma$.

The change made from Game 0 to Game 1 establishes that the parameters associated with this protocol instance - name, $n,\left\{u_{j}\right\},\left\{m_{i j}\right\}$, and $\left\{\sigma_{i}\right\}$ - were generated by the simulator as part of a St query; otherwise, execution would have already aborted. This means that these parameters were generated according to the simulator's procedure described above. Now, dividing the verification equation for the forged signature $\sigma^{\prime}$ by the verification equation for the expected signature $\sigma$, we obtain

$$
\left(\sigma^{\prime} / \sigma\right)^{e}=\prod_{j=1}^{s} u_{j}^{\Delta \mu_{j}}=\left[\prod_{j=1}^{s}\left(g_{j}^{e}\right)^{\beta_{j} \Delta \mu_{j}}\right] \cdot y^{\sum_{j=1}^{s} \beta_{j} \Delta \mu_{j}}
$$

rearranging terms yields

$$
\left[\left(\sigma^{\prime} / \sigma\right) \cdot \prod_{j=1}^{s} g_{j}^{\beta_{j} \Delta \mu_{j}}\right]^{e}=y^{\sum_{j=1}^{s} \beta_{j} \Delta \mu_{j}} ;
$$

Now, provided that $\operatorname{gcd}\left(e, \sum_{j=1}^{s} \beta_{j} \Delta \mu_{j}\right)=1$, we can compute, using Lemma 6.1, a value $x$ from (2) such that $x^{e}=y$.

It remains only to argue that $\operatorname{gcd}\left(e, \sum_{j=1}^{s} \beta_{j} \Delta \mu_{j}\right) \neq 1$ occurs with negligible probability. First, we noted already that not all of $\left\{\Delta \mu_{j}\right\}$ can be zero. Second, the values of $\left\{\beta_{j}\right\}$ are statistically hidden from the adversary ${ }^{25}$ Third, the verification equation checks that each $\mu_{j}^{\prime}$ is in the range $[0, l \cdot N \cdot \max B]$, and each $\mu_{j}$ is also in the same range; thus for each $j$ we have

$$
\left|\Delta \mu_{j}\right|=\left|\mu_{j}^{\prime}-\mu_{j}\right| \leq l \cdot N \cdot \max B<2^{\lceil\lg N\rceil} \cdot 2^{\lceil\lg (l \cdot \max B)\rceil}<2^{2 \lambda_{1}} \cdot 2^{\lambda_{2}}<e,
$$

and since $e$ is prime this means that $\operatorname{gcd}\left(\Delta \mu_{j}, e\right)$ must equal 1. Now, because $e$ is prime, $\operatorname{gcd}\left(e, \sum_{j=1}^{s} \beta_{j} \Delta \mu_{j}\right) \neq 1$ means that $e$ divides $\sum_{j=1}^{s} \beta_{j} \Delta \mu_{j}$, i.e., that $\sum_{j=1}^{s} \beta_{j} \Delta \mu_{j} \equiv 0$

\footnotetext{
${ }^{25}$ Hidden because they are used to compute only the values $\left\{u_{j}\right\}$ in the adversary's view, and thus are informationtheoretically hidden by the $\left\{g_{j}^{e}\right\}$ multipliers.
} 
$\bmod e$. For any particular fixed choice of $\left\{\Delta \mu_{j}\right\}$ values, the probability that this happens, over the independent random choices of each $\beta_{j}$ from $\left[1,2^{\lambda}\right]$, is at most $2^{-\lambda}$, which is negligible. (Let $j^{*}$ be some index such that $\Delta \mu_{j^{*}} \neq 0$ and fix $\left\{\beta_{j}\right\}_{j \neq j^{*}}$. Then let $c \equiv \sum_{j \neq j^{*}} \beta_{j} \Delta \mu_{j} \bmod e$; then $\sum_{j=1}^{s} \beta_{j} \Delta \mu_{j} \equiv c+\beta_{j^{*}} \Delta \mu_{j^{*}}$; and this is congruent to 0 modulo $e$ for exactly one value of $\beta_{j^{*}}$, namely $\beta_{j^{*}}=-(c)\left(\Delta \mu_{j^{*}}^{-1}\right) \bmod e$; since $\beta_{j^{*}}$ is drawn from the range $\left[1,2^{\lambda}\right]$, the probability that it takes on this value is at most $1 / 2^{\lambda}$.)

Thus if there is a nonnegligible difference between the adversary's probabilities of success in Games 1 and 2, we can construct a simulator that uses the adversary to solve the RSA problem, as required.

Wrapping up. Assuming the signature scheme used for file tags is secure, and that the RSA problem with large public exponent is hard, we see that any adversary that wins the soundness game against our public-verification scheme responds in proof-of-storage protocol instance with values $\left\{\mu_{j}\right\}$ and $\sigma$ that are computed according to Pub.P , which completes the proof of Theorem 6.2.

\subsection{Part-Two and Part Three Proofs}

It is easy to see that the Part-Two proof of Section 4.2 carries over unchanged to the case where blocks are drawn from $\mathbb{Z}_{N}$ instead of $\mathbb{Z}_{p}$. All the matrix operations require is that inversion be efficiently computable, and this is, of course, the case in $\mathbb{Z}_{N}$ using Euclid's algorithm, provided we never encounter values in $\mathbb{Z}_{N} \backslash \mathbb{Z}_{N}^{*}$; but such a value would allow us to factor $N$, so they occur with negligible probability provided the RSA problem - and therefore factoring - is hard.

Similarly, erasure decoding works just as well when blocks are drawn from $\mathbb{Z}_{N}$; and because nothing in the proof requires that blocks be distributed uniformly in all of $\mathbb{Z}_{N}$, we could treat each $m_{i j}$ as an element of $\mathbb{Z}_{p_{0}}^{t}$ where $p_{0}$ is some prime convenient for whatever erasure code we employ and $t$ is the largest integer such that $p_{0}^{t}<N$.

\section{Acknowledgements}

We thank Dan Boneh, Guy Rothblum, and Moni Naor for helpful discussions about this work; Eric Rescorla for detailed comments on the manuscript; Giuseppe Ateniese for his suggested improvements to our RSA construction; attendees of the MIT Cryptography and Information Security Seminar and the UC Irvine Crypto Seminar for their questions and comments; and the anonymous Crypto and Asiacrypt reviewers.

\section{References}

[1] M. Aigner and G. Ziegler. Proofs from The Book. Springer-Verlag, 3rd edition, 2004.

[2] N. Alon and M. Luby. A linear time erasure-resilient code with nearly optimal recovery. IEEE Trans. Info. Theory, 42(6):1732-6, Nov. 1996.

[3] G. Ateniese, R. Burns, R. Curtmola, J. Herring, L. Kissner, Z. Peterson, and D. Song. Provable data possession at untrusted stores. In S. De Capitani di Vimercati and P. Syverson, editors, Proceedings of CCS 2007, pages 598-609. ACM Press, Oct. 2007. 
[4] G. Ateniese, R. Burns, R. Curtmola, J. Herring, L. Kissner, Z. Peterson, and D. Song. Provable data possession at untrusted stores. Cryptology ePrint Archive, Report 2007/202, 2007. Online: http://eprint.iacr.org/. Version of 7 Dec. 2007; visited 10 Feb. 2008.

[5] G. Ateniese, R. Di Pietro, L. Mancini, and G. Tsudik. Scalable and efficient provable data possession. In P. Liu and R. Molva, editors, Proceedings of SecureComm 2008, pages 1-10. ICST, Sept. 2008.

[6] P. Barreto and M. Naehrig. Pairing-friendly elliptic curves of prime order. In B. Preneel and S. Tavares, editors, Proceedings of SAC 2005, volume 3897 of LNCS, pages 319-31. SpringerVerlag, 2006.

[7] M. Bellare, O. Goldreich, and A. Mityagin. The power of verification queries in message authentication and authenticated encryption. Cryptology ePrint Archive, Report 2004/309, 2004. http://eprint.iacr.org/,

[8] D. Boneh, B. Lynn, and H. Shacham. Short signatures from the Weil pairing. J. Cryptology, 17(4):297-319, Sept. 2004. Extended abstract in Proceedings of Asiacrypt 2001.

[9] K. D. Bowers, A. Juels, and A. Oprea. Proofs of retrievability: Theory and implementation. Cryptology ePrint Archive, Report 2008/175, 2008. http://eprint.iacr.org/.

[10] H. Cohen. A Course in Computational Algebraic Number Theory, volume 138 of Graduate Texts in Mathematics. Springer-Verlag, 1993.

[11] J.-S. Coron. On the exact security of full domain hash. In M. Bellare, editor, Proceedings of Crypto 2000, volume 1880 of LNCS, pages 229-35. Springer-Verlag, Aug. 2000.

[12] R. Cramer, G. Hanaoka, D. Hofheinz, H. Imai, E. Kiltz, R. Pass, abhi shelat, and V. Vaikuntanathan. Bounded CCA2-secure encryption. In K. Kurosawa, editor, Proceedings of Asiacrypt 200\%, volume 4833 of LNCS, pages 502-18. Springer-Verlag, Dec. 2007.

[13] R. Cramer and V. Shoup. Signature schemes based on the strong RSA assumption. ACM Trans. Info. \& System Security, 3(3):161-85, 2000.

[14] R. Cramer and V. Shoup. Design and analysis of practical public-key encryption schemes secure against adaptive chosen ciphertext attack. SIAM J. Computing, 33(1):167-226, 2003.

[15] Y. Deswarte, J.-J. Quisquater, and A. Saïdane. Remote integrity checking. In S. Jajodia and L. Strous, editors, Proceedings of IICIS 2003, volume 140 of IFIP, pages 1-11. Kluwer Academic, Jan. 2004.

[16] D. Freeman, M. Scott, and E. Teske. A taxonomy of pairing-friendly elliptic curves. Cryptology ePrint Archive, Report 2006/372, 2006. http://eprint.iacr.org/.

[17] D. Gazzoni Filho and P. Barreto. Demonstrating data possession and uncheatable data transfer. Cryptology ePrint Archive, Report 2006/150, 2006. http://eprint.iacr.org/.

[18] L. Guillou and J.-J. Quisquater. A practical zero-knowledge protocol fitted to security microprocessor minimizing both transmission and memory. In C. Günther, editor, Proceedings of Eurocrypt 1988, volume 330 of LNCS, pages 123-8. Springer-Verlag, May 1988. 
[19] S.-H. Heng and K. Kurosawa. $k$-resilient identity-based encryption in the standard model. IEICE Trans. Fundamentals, E89-A.1(1):39-46, Jan. 2006. Originally published at CT-RSA 2004 .

[20] A. Juels and B. Kaliski. PORs: Proofs of retrievability for large files. In S. De Capitani di Vimercati and P. Syverson, editors, Proceedings of CCS 2007, pages 584-97. ACM Press, Oct. 2007. Full version: http://www.rsa.com/rsalabs/staff/bios/ajuels/publications/ pdfs/POR-preprint-August07.pdf.

[21] M. Krohn, M. Freedman, and D. Mazières. On-the-fly verification of rateless erasure codes for efficient content distribution. In D. Wagner and M. Waidner, editors, Proceedings of IEEE Security 8 Privacy 2004, pages 226-40. IEEE Computer Society, May 2004.

[22] M. Lillibridge, S. Elnikety, A. Birrell, M. Burrows, and M. Isard. A cooperative Internet backup scheme. In B. Noble, editor, Proceedings of USENIX Technical 2003, pages 29-41. USENIX, June 2003.

[23] M. Liskov, R. Rivest, and D. Wagner. Tweakable block ciphers. In M. Yung, editor, Proceedings of Crypto 2002, volume 2442 of LNCS, pages 31-46. Springer-Verlag, Aug. 2002.

[24] P. Maymounkov. Online codes. Technical Report TR2002-833, NYU, 2002.

[25] M. Naor and G. Rothblum. The complexity of online memory checking. In E. Tardos, editor, Proceedings of FOCS 2005, pages 573-84. IEEE Computer Society, Oct. 2005.

[26] M. Rabin. Efficient dispersal of information for security, load balancing, and fault tolerance. J. ACM, 36(2):335-48, Apr. 1989.

[27] L. Rizzo. Effective erasure codes for reliable computer communication protocols. ACM SIGCOMM Computer Communication Rev., 27(2):24-36, Apr. 1997.

[28] T. Schwarz and E. Miller. Store, forget, and check: Using algebraic signatures to check remotely administered storage. In M. Ahamad and L. Rodrigues, editors, Proceedings of ICDCS 2006. IEEE Computer Society, July 2006.

[29] M. Shah, R. Swaminathan, and M. Baker. Privacy-preserving audit and extraction of digital contents. Cryptology ePrint Archive, Report 2008/186, 2008. http://eprint.iacr.org/.

\section{A Erasure Codes}

It is easier to verify that a server is storing half the blocks of a file - or any other constant fraction $r$ - than to verify that it is storing all the blocks of a file: probabilistic checks are unlikely to uncover a single file block's being dropped. Before storing it on the server, we would therefore like to encode an $n$-block file into a $2 n$-block file - or, more generally $(n / r)$-block file - with the encoding done in such a way that any $n$ blocks suffice for recovering the original file.

Erasure codes are the codes that provide this property [26, 2]. The parameter $r$ is called the rate. Some erasure codes are rateless in that, for an $n$-block file, they allow the generation of arbitrarily many blocks, any $n$ of which suffice for decoding 26

\footnotetext{
${ }^{26}$ Some erasure codes, called nearly-optimal, require somewhat more than $n$ blocks for decoding: $n(1+\epsilon)$ blocks, where $\epsilon$ is a parameter; a choice of $\epsilon$ has an effect on other code parameters.
} 
An important property of erasure codes is the efficiency of their encoding and decoding procedures. Ideally, one would like both procedures to have performance linear in $n$. This is especially important for our application, where $n$ can be very large. For example, if a block is 1000 bytes and the file being stored is $1 \mathrm{~GB}$, then we have $n \approx 2^{20}$. A code where encoding and decoding take $O\left(n^{2}\right)$ time would be unpleasantly slow.

Another important property of erasure codes is what sort of erasures they can correct. Ideally, we would like the code to correct against arbitrary erasures: any $n$ blocks should suffice for recovering the original file. Some codes, however, correct only against random erasures: any $n$ blocks suffice for decoding with overwhelming probability, whereas an adversarially selected $n$ block set will not suffice.

Unfortunately, no codes are known that provide linear decoding time in the presence of arbitrary erasure. As we show below, we can still make use of codes that correct random erasures only, but in this case additional secret preprocessing is required that makes public retrievability impossible.

\section{A.1 Codes for Public Retrievability}

Traditional Reed-Solomon-style erasure codes can be constructed for arbitrary rates allowing recovery of the original file from any $r$ fraction of the encoded file blocks [27. The encoding and decoding procedures will take $O\left(n^{2}\right)$ time. The code matrix used can be made public and any user can apply the decoding procedure. This provides public retrievability.

We recommend that a systematic code be used: one in which the first $m$ blocks of the encoded file are in fact the encoded file itself. This makes recovering the file from a server that isn't malicious much more efficient: simply ask for the first $m$ blocks.

\section{A.2 Efficient Codes for Private Retrievability}

To employ codes with linear-time encoding and decoding procedures, we will need to take additional measures to transform adversarial erasure to random erasure.

Given a file $M$, encrypt $M$ using a semantically-secure symmetric encryption scheme under a key $k_{\text {enc1 }}$. Now apply the erasure code to the encrypted, permuted file. Assuming the encryption was secure, this is indistinguishable (to any efficient adversary) from applying the erasure code to a random bitstring of the same length as $M$.

Each block in the erasure-encoded file $M^{*}$ depends on some subset of the blocks in the unencoded, encrypted file. Which blocks, precisely, depends on the code family and the particular key used to instantiate a code from that family. Let the unencoded file consist of blocks with indices in the range $[1, m]$, and the encoded file of blocks with indices in the range $[1, n]$. For $i \in[1, n]$, let $\pi_{k}(i) \subseteq[1, m]$ be the pre-encoding blocks on which encoded block $i$ depends for a choice $k$ of code key. Suppose that the erasure code is such that:

1. The distribution of $\pi_{k}(i)$ is independent of the distributions for $\pi_{k}(j)$ for all $j \neq i$. More precisely, the conditional distribution of $\left\{\pi_{k}(i)\right\}_{k}$ over keys $k$ satisfying a specific assignment for $\pi_{k}(j)$ for all $j \neq i$ is statistically indistinguishable from the distribution $\left\{\pi_{k}(i)\right\}_{k}$ over all keys $k$.

2. Each block in the encoded file depends on all blocks in the unencoded file with equal probability. More precisely, for all $a, b \in[1, m]$ and for all $i \in[1, n], \operatorname{Pr}_{k}\left[a \in \pi_{k}(i)\right]=\operatorname{Pr}_{k}\left[b \in \pi_{k}(i)\right]$. 
Such a code would necessarily have a long key, but that key can be generated from a short seed using a secure pseudorandom generator. In fact, it is easy to see that the two properties above are provided by Online Codes [24, 21], a simple nearly-optimal linear-time code.

Now, for any code that provides the two properties above, an adversary could determine which blocks in the encoded file depend on which blocks in the unencoded file only using the contents of the encoded blocks. This is because, ignoring the block contents,

Let the encoded blocks be $\eta_{1}, \ldots, \eta_{n}$. We encrypt each block independently under key $k_{\text {enc2 }}$, using a tweakable block cipher [23], with the tweak for the encryption of $\eta_{i}$ being the block index $i$; the blocks output by this procedure, $m_{1}, \ldots, m_{n}$ are those stored on the server. Assuming the cipher is secure, the contents of these blocks are indistinguishable from random and independent of each other.

It is now clear that, without knowledge of $k_{\text {enc1 } 1}, k$, or $k_{\text {enc2 } 2}$, the encoded file $m_{1}, \ldots, m_{n}$ reveals no information about the code used, and, specifically, about which code blocks depend on which pre-encoding blocks. Thus no adversary that erases blocks can do better than random erasure, which is exactly the property we require for decoding to work with overwhelming probability.

\section{B Can We Eliminate $B$ Coefficients?}

In both schemes proposed in Section 3 , the verifier sends with each index $i$ of a query a coefficient $\nu_{i}$ from a set $B$. If we could avoid sending these coefficients - equivalently, if we could set $B=\{1\}$, then we would obtain a scheme that is more efficient in several respects:

- the verifier would need to flip fewer coins in generating a query;

- the query would be shorter by $l \cdot \lg \# B$ bits;

- the prover's computation would be greatly reduced: essentially, one multiplication instead of $l+1$ multiplications in the first scheme; one exponentiation instead of $l+1$ exponentiations in the second scheme ${ }^{27}$, and

- the verifier's computation would also be reduced, though not so dramatically.

Unfortunately, it is clear from Lemma 4.5 that the proof techniques of Section 4.2 cannot apply when $\# B=1$, since we will not then have $\epsilon>1 / \# B$, however large the adversary's success probability $\epsilon$ is.

This is not just a proof problem. Below, we present an attack on the schemes of Section 3 when $B=\{1\}$. In the attack, the server stores $n-1$ blocks instead of $n$, can answer a nonnegligible fraction of all queries, yet no extraction technique can recover any of the original blocks.

A note on notation. In this section, we will make some simplifications to the notation for the sake of brevity and clarity. First, obseve that the Part-1 proofs of Section 4.1 do apply in the case that $B=\{1\}$. We will thus elide the authenticators $\left\{\sigma_{i}\right\}$ in our attack; this allows us to address both the scheme with private verification and the scheme with public verification simultaneously. Second, we will set the number of sectors per block, $s$, to 1. Our attack easily generalizes to the case $s>1$, but this simplification allows us to eliminate a subscript and simplify the presentation.

\footnotetext{
${ }^{27}$ The $l$ element summation computed by the prover in the first scheme requires work comparable to a multiplication when $l \approx \lg p$.
} 
The attack. With the simplifications above, consider an $n$-block file with blocks $\left(m_{1}, \ldots, m_{n}\right)$. A query will consist of $l$ indices $I \subset[1, n]$; the response will be $\mu=\sum_{i \in I} m_{i}$. We assume that $l$ is even 28

The adverary chooses an index $i^{*}$ at random from $[1, n]$. For each $i \neq i^{*}$, he chooses $\tau_{i} \stackrel{\mathrm{R}}{\leftarrow}$ $\{-1,+1\}$ and sets

$$
m_{i}^{\prime} \leftarrow m_{i}+\tau_{i} m_{i^{*}}
$$

Now the adversary remembers $\left(m_{1}^{\prime}, \ldots, m_{i^{*}-1}^{\prime}, m_{i^{*}+1}^{\prime}, m_{n}^{\prime}\right)$. Clearly, the adversary needs to store one less block than an honest server would.

Now, consider a query $I$. If $i^{*} \notin I$, the adversary responds with $\mu^{\prime}=\sum_{i \in I} m_{i}^{\prime}$. Otherwise, $i^{*} \in I$, and the adversary responds with $\mu^{\prime}=\sum_{i \in I \backslash\left\{i^{*}\right\}} m_{i}^{\prime}$.

In our analysis, we will use the following simple lemma:

Lemma B.1 ([1], p. 12). For $k \geq 2$ we have $\left(\begin{array}{c}k \\ \lfloor k / 2\rfloor\end{array}\right) \geq 2^{k} / k$.

Proof. $\left(\begin{array}{c}k \\ \lfloor k / 2\rfloor\end{array}\right)$ is the largest of the $k$ values $\left(\begin{array}{l}k \\ 1\end{array}\right),\left(\begin{array}{l}k \\ 2\end{array}\right), \ldots,\left(\begin{array}{c}k \\ k-1\end{array}\right)$, and $\left(\begin{array}{l}k \\ 0\end{array}\right)+\left(\begin{array}{l}k \\ l\end{array}\right)$; and so it must be at least as large as their average, $2^{k} / k$.

In the case $i^{*} \notin I, \mu^{\prime}$ will be correct provided that we have $\sum_{i \in I} \tau_{i}=0$. But this happens when the number of $+1 \mathrm{~s}$ and the number of $-1 \mathrm{~s}$ are equal in $\left\{\tau_{i}\right\}_{i \in I}$, and this happens with probability

$$
\left(\begin{array}{c}
l \\
l / 2
\end{array}\right) \cdot\left(\frac{1}{2}\right)^{l / 2} \cdot\left(\frac{1}{2}\right)^{l / 2} \geq\left(\frac{2^{l}}{l}\right) \cdot\left(\frac{1}{2^{l}}\right)=\frac{1}{l} .
$$

In the case $i^{*} \in I, \mu^{\prime}$ will be correct provided we have $\sum_{i \in I \backslash\left\{i^{*}\right\}} \tau_{i}=1$. This happens when there are $l / 2-1-1 \mathrm{~s}$ and $l+1 \mathrm{~s}$ in $\left\{\tau_{i}\right\}_{i \in I \backslash\left\{i^{*}\right\}}$, and this happens with probability

$$
\left(\begin{array}{c}
l-1 \\
l / 2-1
\end{array}\right) \cdot\left(\frac{1}{2}\right)^{l / 2-1} \cdot\left(\frac{1}{2}\right)^{l / 2}=\left(\begin{array}{c}
l-1 \\
\lfloor(l-1) / 2\rfloor
\end{array}\right) \cdot\left(\frac{1}{2}\right)^{l-1} \geq\left(\frac{2^{l-1}}{l-1}\right) \cdot\left(\frac{1}{2^{l-1}}\right)=\frac{1}{l-1}>\frac{1}{l} .
$$

Thus the adversary can respond to $1 / l$ fraction of queries where $i^{*} \in I$ and to a $1 / l$ fraction of queries where $i^{*} \notin I$; so he can respond to a $1 / l$ fraction of all queries, which is clearly nonnegligible.

But now it is impossible for any extraction strategy to recover any block, let alone a $\rho$ fraction of all blocks. This is because the subspace known to the adverary is insufficient to determine any block. Indeed, the adversary's knowledge is consistent with any value for any block. Fix $\left(m_{1}^{\prime}, \ldots, m_{i^{*}-1}^{\prime}\right.$, $\left.m_{i^{*}+1}^{\prime}, m_{n}^{\prime}\right)$ where $m_{i}^{\prime}=m_{i}+\tau_{i} m_{i^{*}}$. Suppose we believe that $m_{i^{*}}=a$ for any value $a$. This fixes $m_{i}$ for each $i \neq i^{*}$, as $m_{i}=m_{i}^{\prime}-\tau_{i} m_{i^{*}}$. If we believe, for some index $\lambda \neq i^{*}, m_{\lambda}=a$, then $m_{i^{*}}$ is fixed because $m_{\lambda}^{\prime}=m_{\lambda}+\tau_{\lambda} m_{i^{*}}$ implies $m_{i^{*}}=\left(\tau_{\lambda}\right)\left(m_{\lambda}^{\prime}-a\right)$, and the argument proceeds as before. Since the adversary's knowledge is consistent with any choice of value for any (single) block, it cannot be the case that it allows recovery of the value of any block.

\section{Extensions}

In this section we discuss a few possible extensions to our scheme.

\footnotetext{
${ }^{28}$ If $l$ is odd, the adversary can set $m_{i}^{\prime} \leftarrow m_{i}+\left(l^{-1} \bmod p\right)\left(m_{i^{*}}\right)$, which allows him to respond to that $l / n$ fraction of queries where $i^{*} \in I$.
} 
Towards adaptively adding storage blocks. In our current scheme the storage algorithm takes as input a whole file $M$ before storing the data and publishing the random name value. In some situations it might make sense to be able to incrementally append blocks to the end of the file. For example, we might want to append to the file itself.

Our current proof relies upon the simulator being able to "program" the random oracle so that it can sign the blocks of $M$. The simulator doesn't not give out the name value until it learns the whole file $M$ value so that the attacker cannot query the random oracle until the simulator knows how to program it. However, if we want to allow for appending the simulator will need to disclose name before learning some values of the block values.

We can overcome this issue by adding a bit of randomness to our system. In addition, to storing $s$ sectors per data block the storage algorithm will additionally store one more element that is chosen randomly in $\mathbb{Z}_{p}$. The storage, proof, and verification algorithms will behave just as though there were $s+1$ sectors stored. In the simulation, the simulator will set the program the oracle so that it can sign a "random" block, and when it gets a request to store a specific block it will choose the randomness of the last "dummy" sector such that it can sign the entire block. Our techniques are similar to those used by Coron [11] for tight proofs of signature schemes.

We note that these techniques are useful for validating the integrity of blocks appended to the end, however, in a complete scheme we still need to concern ourselves with how to redundantly encode the file such that blocks can be appended to the end.

Towards removing random oracles. One interesting question is whether we can realize our scheme in the standard model (without random oracles). One step towards this direction is to use a common random string consisting of $n_{\text {Max }}$ group elements $h_{1}, \ldots, h_{n_{\text {Max }}}$, where $n_{\text {Max }}$ is the maximum number of blocks used in a file. In this situation, we could replace the call to $H$ (name $\| i$ ) with $h_{i}$. In addition, the simulator would use the techniques from the paragraph above so that it could "program" the $h$ values for signing a "random" message and later use the extra sector to make the proof go through. 